\title{
Percutaneous Management of Portal Vein Patency and Integrity Problems - Catheter Directed Local Thrombolysis, Stenting, Endoluminal RFA \& Angioplasty by Balloon or Stenting
}

\author{
M. Mizandari ${ }^{1, *}$, N. Habib ${ }^{2}$ \\ ${ }^{1}$ High Technology Medical Center - University Clinic, Georgia \\ ${ }^{2}$ Department of Biosurgery \& Surgical Technology, Faculty of Surgery, \\ Oncology, Reproductive Biology \& Anaesthetics, Imperial College, UK
}

Copyright@2016 by authors, all rights reserved. Authors agree that this article remains permanently open access under the terms of the Creative Commons Attribution License 4.0 International License

\begin{abstract}
Purpose: Spectrum of PV patency and integrity percutaneous restoration techniques is presented. Material and methods: Total twenty patients with portal hypertension due to PV patency \& integrity problem; among them percutaneous recanalization was attempted in fifteen patients with PV tumor thrombus using a novel endoluminal bipolar radiofrequency device. The procedure was completed in twelve of fifteen RFA cases; RFA was followed by balloon angioplasty (six cases) or vascular stent placement (six cases). In two cases catheter directed local thrombolysis was performed to acutely manifest fresh PV thrombosis, caused by thrombophylia and HCC. In two cases of pancreatitis induced PV thrombosis \& stricture and one case of pancreatitis induced PV thrombosis \& stricture and porto-biliary fistula, PV stenting was performed. The PV tributary was percutaneously accessed under US guidance and 5G guide catheter was manipulated through the obstruction using guidewire technique under DSA guidance. In case of thrombolysis thrombolytic agent was injected directly distal to the thrombus, the stenting procedure was completed by self-expanding vascular stent placement. Results: The technical success rate was $85.0 \%$; in three cases $(15.0 \%)$ wire conduction through the organized thrombus was impossible. Posprocedure portography documented significantly improved portal vein blood flow in all patients, to whom the procedure was completed. Porto-biliary fistula was successfully managed by percutaneous stenting. In three cases post procedure bleeding was documented, which led to multiorgan failure and death in one case. Conclusions: The management of PV patency and integrity problems by percutaneous stenting or endoluminal RFA and stenting is an effective technique; it should be suggested as a treatment option for otherwise incurable patients and might be used as a bridge for further treatment. Post-procedure intraperitoneal bleeding is a possible life-threatening complication which
\end{abstract}

should be prevented by procedure track ablation or embolization.

Keywords Portal Vein, Thrombosis, Recanalization, Endoluminal RFA, Stenting

\section{Introduction}

Portal vein thrombosis is being increasingly recognized not only in cirrhotic and malignant conditions like hepatocellular carcinoma, but in non-cirrhotic patients also. [1]. In patients with liver cancer portal vein thrombus (PVT) is a relatively common, but difficult, condition to manage. It often indicates an advanced stage of disease with poor prognosis and is contraindicated for surgical thrombectomy $[2,3]$. Liver parenchyma normally receives $70 \%$ of its blood from the portal vein [4,5]. As a result of PVT, a decrease in partial blood supply to the affected segments could occur, which may lead to further deterioration of liver function. Currently, there is no effective modality available to treat this condition. Percutaneous transhepatic placement of portal vein stent as a possible treatment option for PV hypertension symptoms relief in patients with recurrent perihilar biliary malignancy has been reported (6). It should be mentioned, that a direct access to PV might be performed using transjugular and transsplecnis routes $(7,8,9)$, although it should be mentioned, that transsplenic access is associated with relatively high risk of intrasplenic or intraperitoneal bleeding. An endovascular bipolar radiofrequency (RF) catheter has been developed for percutaneous ablation of tumor thrombus (HabibTM VesOpen, EMcision Limited, London, UK). Initial animal studies showed that the device could be used safely for vascular remodeling with increase in 
the luminal diameter and flow [10].

SMV, SV and PV thrombosis is not common, but not very rare problem in non-oncology patients also. Such pathologies, as thrombophilic conditions, pancreatitis also major abdominal surgery and repetitive abdominal trauma may cause SMV and PV thrombosis requiring percutaneous thrombectomy and thrombolysis and vascular stent placement $(11,12,13)$.

Besides PV patency problem due to thrombus formation, we may face PV integrity problem also, when pancreatico-portal or bilio-portal fistula is formed as a complication of pancreatitis or PTC procedure; portal vein stent-graft placement either in bile duct or in PV branch is the possible treatment option reported in porto-biliary fistula cases $(5,10)$.

We report clinical application of percutaneous imaging guided management, including use of endoluminal RFA device for mainly oncology, but also non-oncology cohort of twenty patients with PV patency and integrity problems. Stent positioning and catheter directed local thrombolysis is accepted technique for PV patency restoration; the RF processing is also widely used technique of malignant neoplasms treatment. Novel is the use of endoluminal RF device for inoperable PV malignant thrombus ablation; the use of this technique for otherwise untreatable PVT management was approved by "High Technology Medical Center- University Clinic" ethical committee

\section{Material and Methods}

Twenty patients with portal hypertension due to PV patency (among them - one patient with PV integrity problem also, due to porto-biliary fistula formation) underwent the percutaneous imaging guided management in the period from Dec 2010 to May 2015. The cohort included 11 male and 9 female patients; age varied from 45 to 79 . All patients underwent US and multiphase contrast CT examinations. In fourteen patients with PV tumor thrombus and one case of cirrhosis percutaneous recanalization using a novel endovascular bipolar radiofrequency device was attempted; the procedure was completed in twelve cases; In all completed oncology and cirrhosis cases RFA was followed by balloon angioplasty (six cases; five - HCC, one-retroperitoneal sarcoma) or vascular stent placement (six cases; five - HCC, one - liver cirrhosis). All oncology patients were receiving the supportive care only, as they were not considered the candidates of any curative treatment. In two cases catheter directed local thrombolysis was performed to clinically manifest fresh PV thrombosis, caused by thrombophylia and HCC. In two cases of pancreatitis induced PV thrombosis \& stricture and one case of pancreatitis induced PV thrombosis \& stricture with porto-biliary fistula PV stenting was performed. Portal thrombosis was located in the right branch (2 patients), left branch (1 patient), right and main portal vein (5) left and main portal vein (4) main portal vein (7 patients); in 3 of 16 patients with main PV thrombosis the thrombus extended into SMV and in 1 case - in SV. The processed thrombus extent varied from 2 to $8 \mathrm{~cm}$. Disease clinical manifestation duration varied from 0 days (1 patient with thrombophylia and 1 patient with HCC) to 6 months. Patient selection for PV percutaneous transhepatic intervention was based on clinical findings manifested by pain only (local thrombolysis cases) or pain and liver function severe deterioration in the rest cases, caused by PVT as documented by radiological data. The endoluminal RFA device has been described elsewhere [10]. In brief, the HabibTM VesOpen is a CE marked; endovascular RF catheter, $110 \mathrm{~cm}$ in length, available in $5 \mathrm{Fr}$ or $8 \mathrm{Fr}$. The catheter has two stainless steel electrodes at the tip and can be used with 2-50 W of power generated by an appropriate RF generator. It can be used in monopolar or bipolar mode. In monopolar mode, high-frequency current passes between the electrodes and grounding pad (patient electrode) applied to the patient. In bipolar mode, the high-frequency current passes between the two electrodes. Under sedation and local anesthesia of $20 \mathrm{ml}$ of $0.5 \%$ Marcain (bupivacaine hydrochloride), the portal vein tributary was percutaneously accessed under US guidance; depending on the thrombus location right or left portal vein branch was approached through the subcostal or intercostal space. An $18 \mathrm{G}$ puncture needle accommodating a 0.035 -inch guidewire was inserted into the portal vein under the guidance of real-time ultrasound using a "Free Hand" or fixed needle guide technique. Following percutaneous puncture of the liver, $10 \mathrm{ml}$ of Omnipaque (Iohexol) contrast media was injected under digital subtraction angiography (DSA) for visualization of the portal system proximal to the thrombus to confirm the position of the needle and to guide the subsequent procedures.

Once the puncture needle was in the correct position, it was removed after placement of a 0.035 -inch guidewire into the portal vein. Using this guidewire, a $5 \mathrm{Fr}$ guide catheter was introduced into the portal vein and was manipulated through the thrombus. The position was confirmed by portography, performed the guiding catheter, having it's tip positioned distal to the thrombus. In thrombolysis cases 100 $\mathrm{mg}$ of Actilise was injected directly distally from the thrombus; in the stenting cases procedure was completed by self-expanding vascular stent placement according the 0.035 inch diameter guidewire; for RFA processing the endoluminal radiofrequency device was inserted into the thrombus according the guidewire (5 $\mathrm{Fr}$ device requires 0.014 inch and $8 \mathrm{Fr}$ device -0.035 inch diameter guidewire); The VesOpen device was connected to a RF generator (RITA Medical Angiodynamics, USA) and 10 to $15 \mathrm{~W}$ of RF energy was applied for 2 min. Immediately after RF application angioplasty by balloon inflation or stent placement was performed. In balloon angioplasty cases 9-10 $\mathrm{mm}$ diameter balloon (Delivery device diameter 5F, Cook Medical, USA) was used. The balloon was inflated for $2 \mathrm{~min}$. In cases of RFA \& stenting RFA processing was performed by 5 or $8 \mathrm{Fr}$ endoluminal device. The stent angioplasty was performed by $14 \mathrm{~mm}$ diameter self-expanding nitinol 
vascular stent (COOK medical) positioning in the PV processed segment so that the positioned stent extends proximal and distal from the blocked segment at least 10-15 $\mathrm{mm}$. Portography after stent positioning was performed to document the restored patency. In 4 recent cases of PV RFA processing and stenting after final portography the procedure was finished by track RFA ablation (3 cases) and coil placement (1 patient); it has been started after having posprocedure bleeding complications. The puncture site was covered with a seff-adherent adhesive dressing. After stable observation overnight, the patients were discharged home from the hospital, except the patients with postprocedure bleeding. No portal pressure measurements were made in this cohort of patients. US follow up was performed in 1,3 and 6 weeks initially and monthly after this. CT follow up was performed in 3, 6 and 12 months after the procedure

\section{Results}

As we do not aim to evaluate the immunological aspects of $\mathrm{RF}$ application, results of $\mathrm{PV}$ flow restoration in both benign and malignant conditions are discussed in one group; in one case of pancreatitis induced PV integrity problem porto-biliary fistula was successfully managed by stenting. The procedure technical success rate was $85.0 \%$; in three cases (15.0\% - all in endoportal RFA group) procedure failed as the wire conduction through the organized thrombus was impossible. In cases of RFA endoluminal processing and balloon angioplasty portography revealed an immediate improvement of blood flow into hepatic parenchyma through the portal vein. Patients tolerated the procedure well.

After RFA \& stenting patients showed complete or almost complete recanalization of PV obstructed segment, no intraprocedural complications were detected. PV patency duration after recanalization by endoportal RFA \& stenting varied from 1 week to 22 months. Positive clinical result as shown by clinical symptoms (pain, fever), liver functional tests and patient's condition improvement in 6 weeks after the recanalization was achieved in $9(52.9 \%)$ patients (including one thrombolysis patient with HCC induced thrombus, who generated rethrombosis in 3 weeks) of 17 , to whom procedure was completed; Rethrombosis in the period from 1 to 6 weeks was documented in $6(50.0 \%)$ of $12 \mathrm{PV}$ RFA patients, to whom the procedure was completed; among them - in 4 RFA \& balloon dilatation cases, 1 RFA and stenting patient and 1 post-thrombolysis HCC patient. In one case of RFA \& stenting the patient underwent the successful TACE procedure

In three cases (all after 8 Fr RFA device application) postprocedure bleeding was documented which was self-limiting in one case, was managed conservatively by the peritoneal cavity drainage and hemostatic infusion in the second; in the third case peritoneal drain showed the continuing bleeding, finally leading to polyorganic failure and death as the patient refused to undergo the surgery (grade 1,2 and 4 by CTCAE respectively). Bleeding was prevented in all cases when track ablation or track coiling was performed. Postprocedure portography documented significantly improved portal vein blood flow in both thrombolysis patients, both PV stenting patients and patient with PV thrombosis and porto-biliary fistula; in porto-biliary fistula case the PV integrity was also restored. In pancreatitis induced two PV thrombosis cases concomitant SMV thrombosis remained unchanged.

\section{Discussion}

Although transarterial chemoembolization (TACE) and systemic chemotherapy are standard methods for management of advanced stage HCC [2-5], few effective treatment options are available for dealing with intravascular tumor spread. An endovascular RF catheter is described, which was used safely to recanalize (partially or completely) the thrombosed portal vein in conjunction with balloon dilatation or stent placement after RF to the thrombus. In following endoportal RFA and balloon dilatation, tumor thrombus was still seen but smaller in size with concomitant increase in portal flow as demonstrated by portal venography and duplex Doppler scan. During RF, the energy passes between the electrodes and biological tissues to cause coagulation of a selected area. The pathophysiology of this phenomenon is that the high-frequency alternating electric current applied through the electrodes results in rapid movement of intracellular ions in opposite directions. Ionic motion creates frictional forces that generate heat around the electrodes and eventually, around the tissue surrounding the catheter causing the coagulative necrosis of tumor cells. Important point is also the RF generated antitumor immune response, which is not discussed in this paper. After heating the thrombus and vessel wall, the RF catheter is removed immediately and followed by balloon angioplasty or stent placement. The combination of thermal energy and balloon pressure has been proven to effectively remodel intraluminal atherosclerotic plaque and increase lumen diameter [10]. In vivo animal studies also showed that RFA thermal angioplasty could be performed safely and resulted in a greater increase in cross-sectional area in peripheral arteries than nonheated arteries after conventional balloon angioplasty $[16,17]$. This approach has been successful in clearing blocked metal vascular stents, such as TIPS. The RFA processing by $8 \mathrm{Fr}$ diameter endoluminal device, followed by vascular stent placement showed significantly better results having undoubtedly less tendency to early rethrombosis and showing the significant positive clinical impact. So, bigger diameter RFA device enabled to process the thrombus more efficiently; it should be mentioned also, that 0.035 inch diameter wire, which is accepted by $8 \mathrm{Fr}$ devices provides much stronger support for device introducement. Interestingly, no clinical benefit was shown in 2 cases of procedure technical success with no early rethrombosis; retrospective analysis showed the presence of very prominent collateral flow in both cases - we can suggest, 
that already existing collaterals recruit the injured liver resource so fully, that PV reopening could not result in any clinical improvement for that moment.

The theoretical benefit of portal vein thrombus RFA could be potentially fourfold. First, it may destroy the tumor thrombus and prevent or delay widespread dissemination. Second, it may improve liver function and hence patients become the candidates of subsequent TACE, RF ablation, surgery, or chemotherapy. Third, it may lead to reduction of portal hypertension and therefore the likelihood of bleeding oesophageal varices. Fourth, it may rise the antitumor immune response. All of the above have not yet been proven by our pilot group and will be the subject of further study. Recanalization of the portal vein will be more beneficial if it is followed by a therapeutic approach, such as TACE, otherwise, as we suggest, rethrombosis inevitably will occur. At this stage, it is clear that a vascular stent should be placed following RF ablation and the working track should be ablated or embolized to avoid the bleeding, which may be more prominent the better reopening of $\mathrm{PV}$ has been achieved.

Intravascular RFA of tumor thrombus is a novel concept. The diminution of the tumor thrombus is probably due to desiccation of the lesion analogous to RFA of HCC tumors. Because the tumor was not removed from the portal vein for histological verification, it is difficult at this stage to ascertain the level of tumor destruction, whether the tumor was completely or partially destroyed. In the absence of biopsy, it is difficult to be certain that the occlusion was due to tumor thrombus and not venous thrombosis. However, it was highly suggestive as in all cases the tumor was in continuity with the portal venous thrombus.

It is highly suggestive, that recanalization of the portal vein will be more beneficial if it is followed by a therapeutic approach, such as TACE or RFA. Other studies have shown benefit with percutaneous direct intratumoral RF of tumor thrombosis in the portal circulation. Thanos et al. [18] reported direct $\mathrm{RF}$ ablation of portal vein tumor thrombus in continuity with hepatocellular carcinoma and observed patency of the portal vein 16 months after RF ablation. Likewise, Giorgio et al. [19] discussed the merit of percutaneous RF ablation of both the HCC nodule and the portal vein tumor thrombus and concluded that this approach has high efficacy and safety and a low rate of complications. They also observed improved survival following the procedure.

Percutaneous transhepatic access for PV patency and integrity management is much more convenient in comparison with tranjugular and transsplenic routes as it provides with possibility to have much less distance from skin entry point to the area of interest enabling to manipulate on PV easily. Percutaneous management showed positive technical and clinical results in PV thrombosis benign cases; in all of them PV patency partial or complete restoration led to patient's condition improvement; in pancreatitis induced PV thrombosis cases splenic vein thrombosis remained unchanged but it was not clinically important resulting only in spleen moderate enlargement and being compensated by spleno-systemic collateral flow.

Our experience with the portal vein thrombus shows, that in the setting of $\mathrm{HCC}$ and liver failure is multifactorial and PV successful recanalisation is not necessarily followed by clinical improvement; we can suggest that it might be due to preexisting maximal recruitment of the liver parenchyma by collateral flow. Although the relatively high mortality, shown in our cohort patients might be (and, actually already is) prevented by working track obligatory ablation or coiling, the further investigation of technique limitations and procedure indication is warranted.

\section{Conclusions}

Percutaneous imaging guided management of portal vein patency and integrity problems showed to be feasible and effective in non-oncology patients - PV obstructed segment recanalization by percutaneous stent placement is the minimally invasive intervention, enabling to avoid the portal hypertension, improving the liver function and decreasing the risk of variceal bleeding. Portal vein malignant thrombus recanalization by endoportal RFA \& stenting is promising technique, enabling to restore the PV flow in otherwise incurable patients and should be considered as an independent treatment option or as a bridge to transarterial embolization, converting those patients from supportive to curative treatment group. Postablation angioplasty should be performed by stenting rather than by balloon dilatation as it shows to have less rethrombosis rate. When followed by track ablation and/or embolization the procedure is safe and might be suggested routinely as a treatment option in selected patients. Larger study is needed to assess any potential clinical usefulness of intravascular RFA, including the possible antitumor immune response.

\section{Abbreviations}

PV - portal vein, HCC - hepatocelullar carcinoma, RFA radiofrequency ablation, DSA - digital subtractive angiography, PVT - portal vein thrombosis, SMV - superior mesenteric vein, SV - splenic vein, TACE - transarterial chemoembolization 


\section{Appendix}

Figures 1-14. Patient M. Left liver lobe tumor induced main portal vein (MPV) thrombus recanalization by endoportal RFA \& stenting

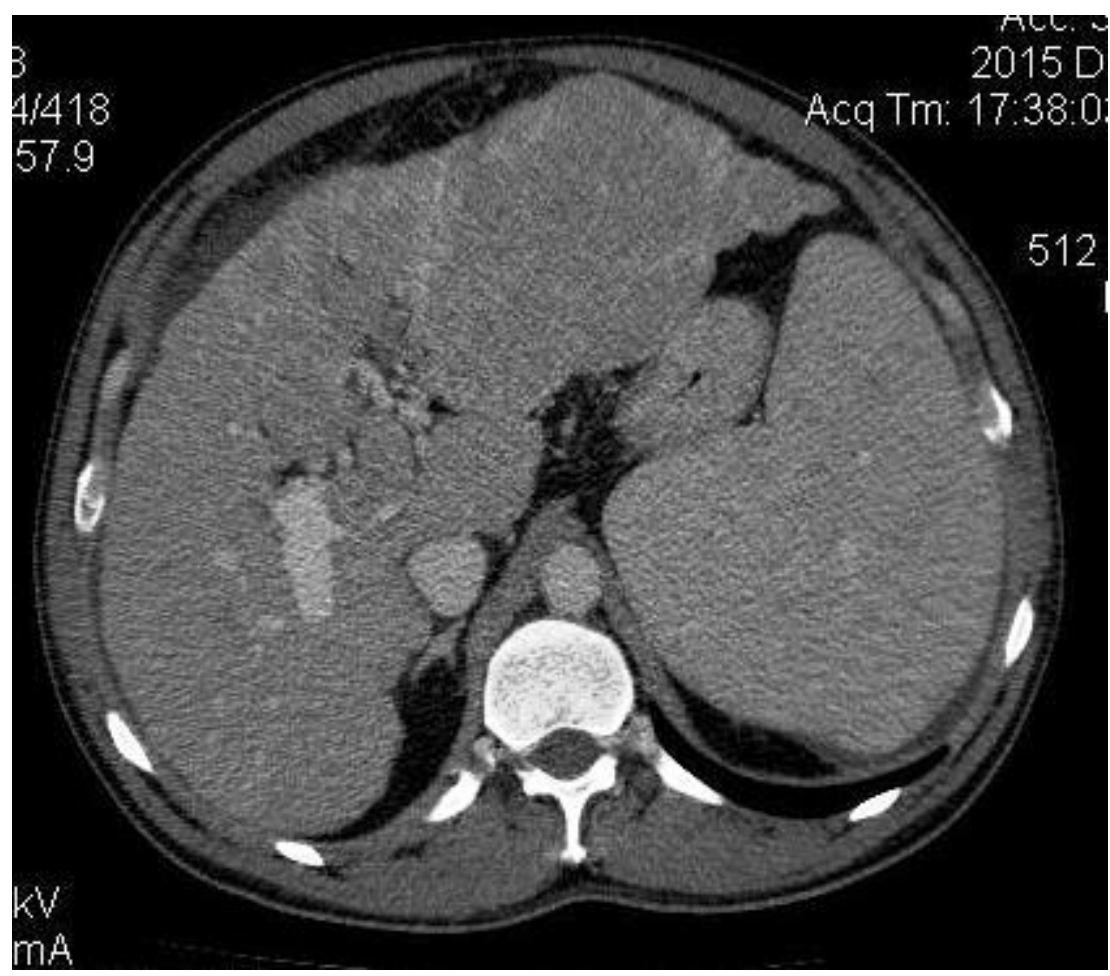

Figure 1. CT, portal phase. Giant left lobe mass and MPV tumor thrombus is seen

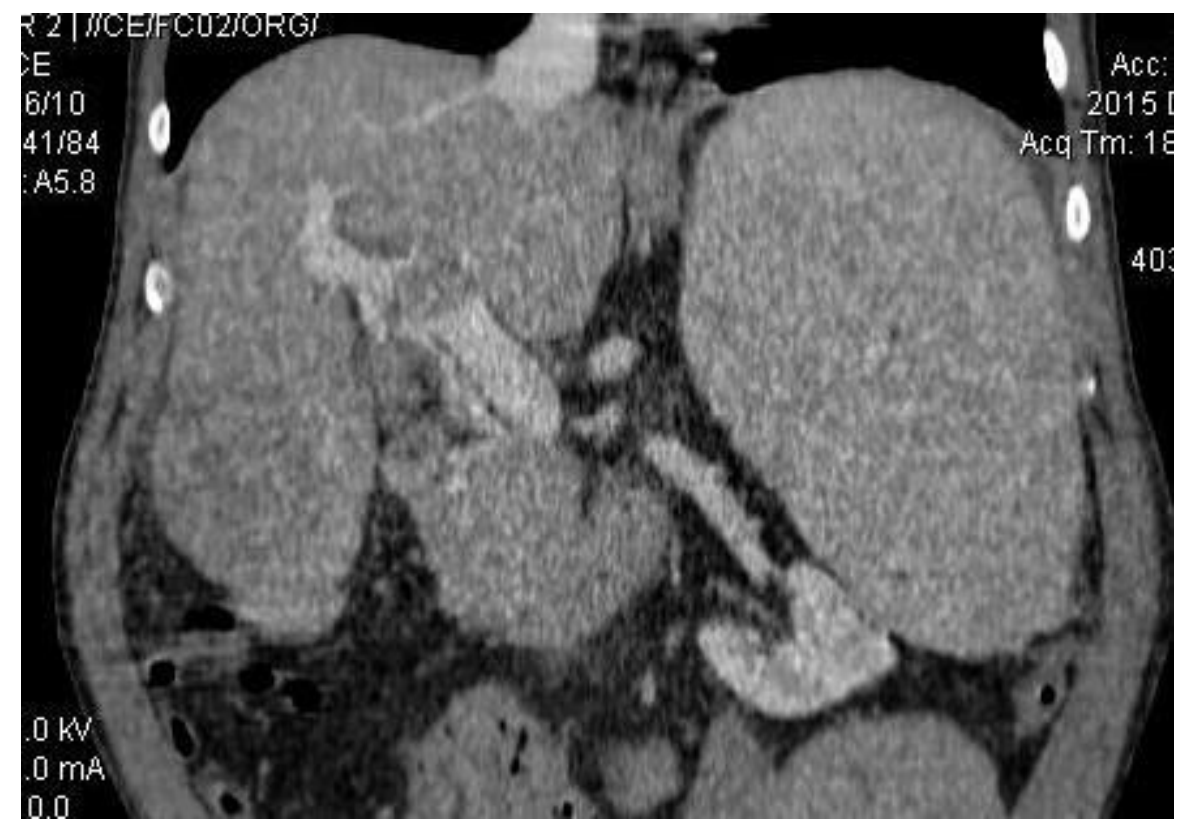

Figure 2. CT, portal phase, coronal reconstruction. MPV tumor thrombus. 

Thrombolysis, Stenting, Endoluminal RFA \& Angioplasty by Balloon or Stenting

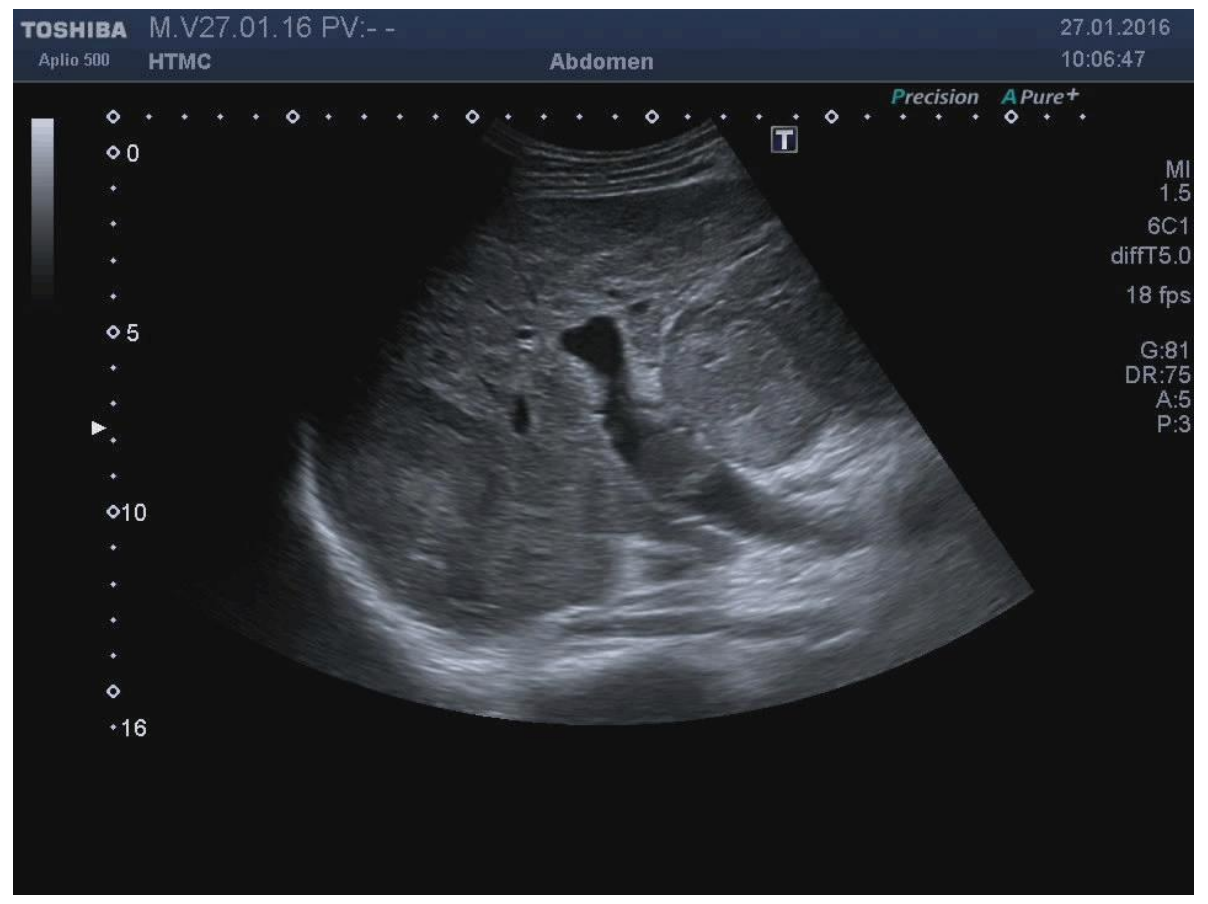

Figure 3. US shows the MPV thrombus

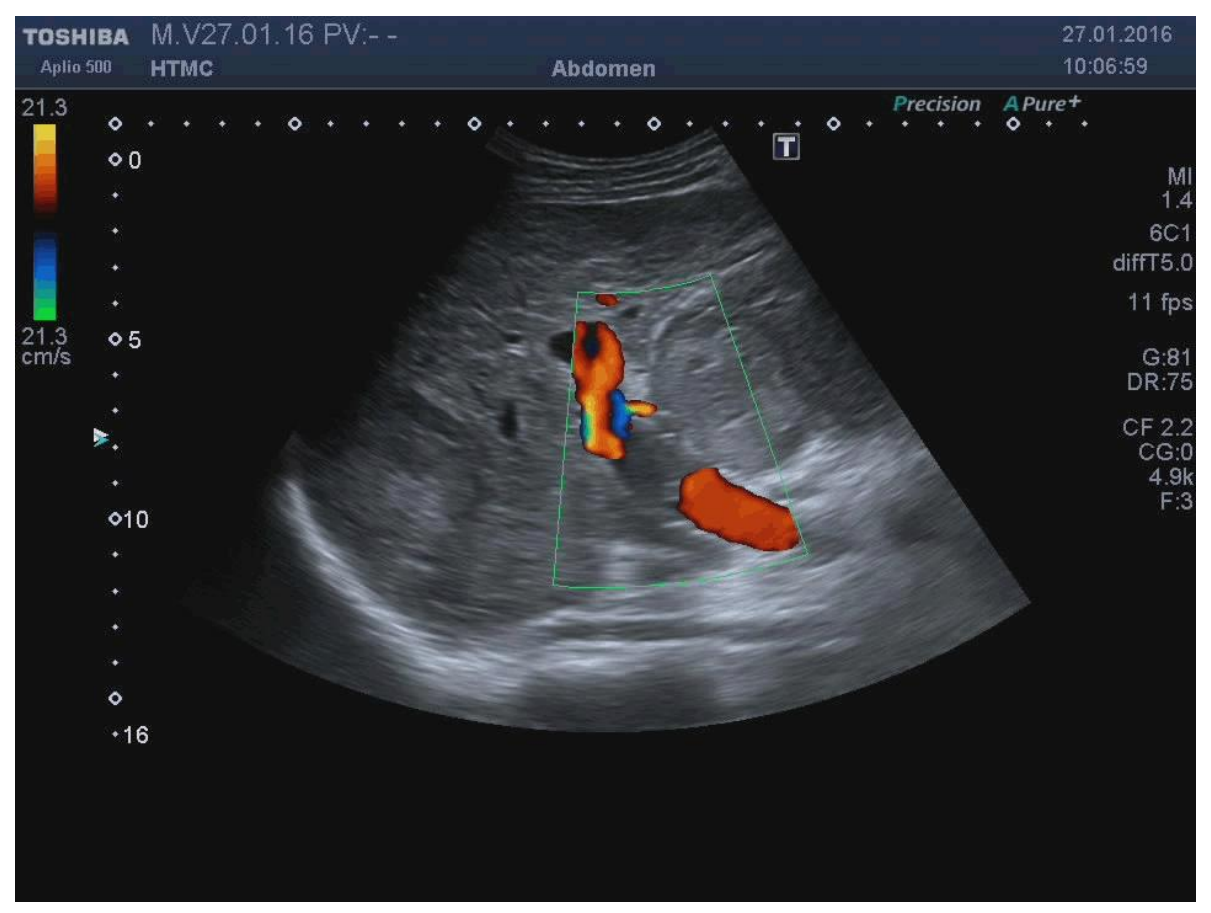

Figure 4. US with color Doppler outlines the MPV thrombus 


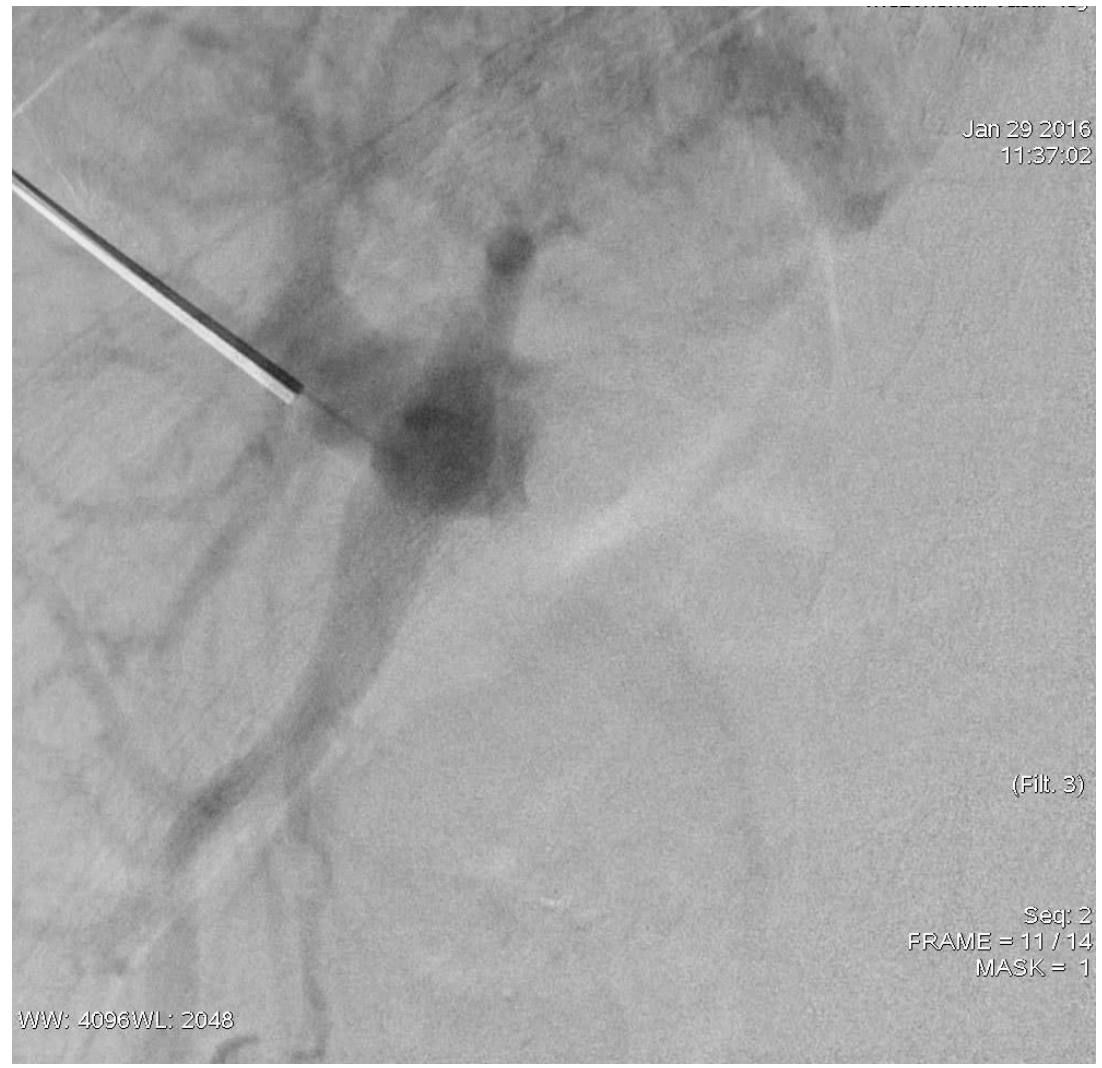

Figure 5. VesOpen procedure. Portography - contrast is injected by $18 \mathrm{G}$ needle, positioned using US guided transhepatic approach; thrombus "upper" margin is outlined

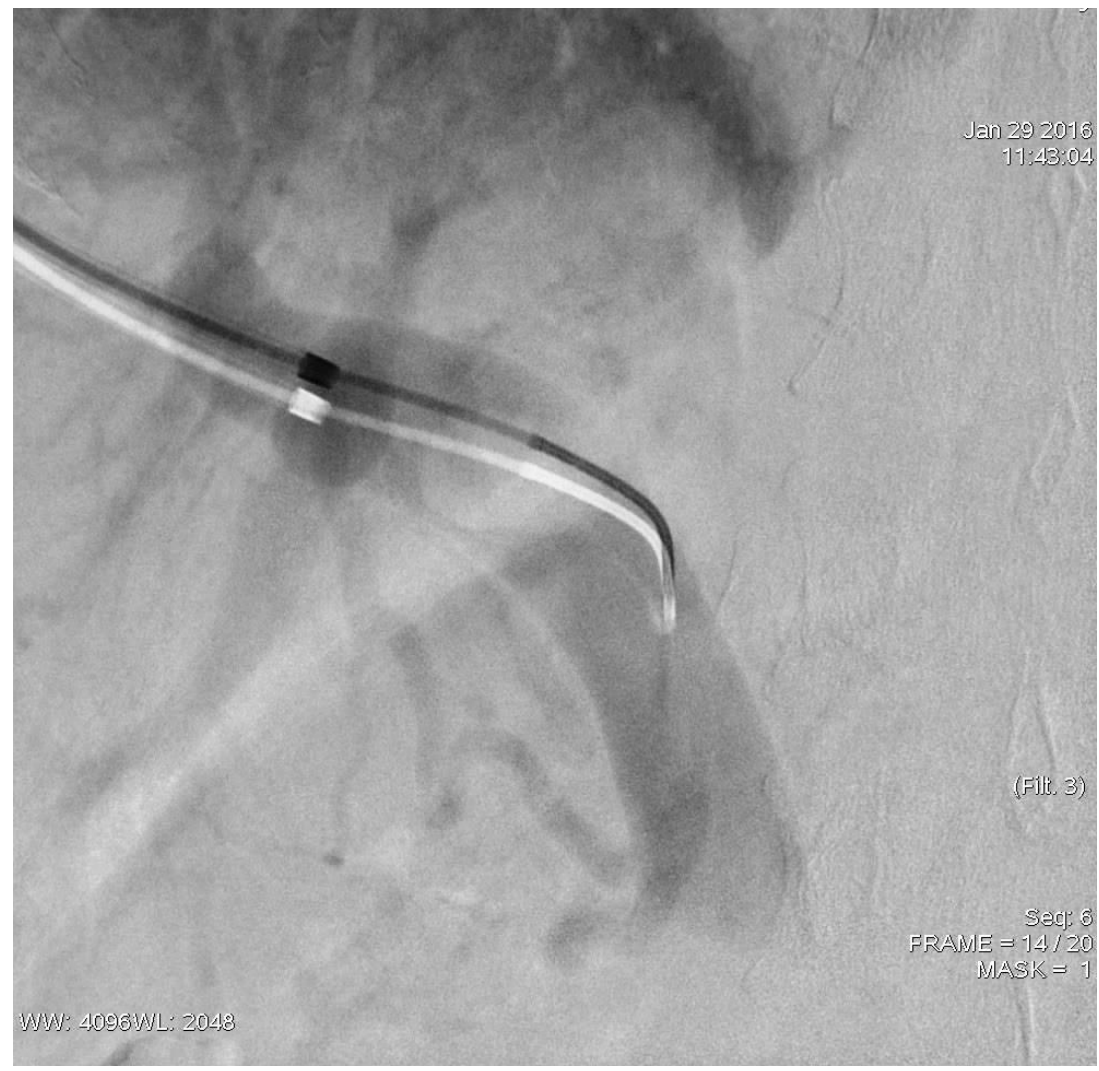

Figure 6. VesOpen procedure. Portography - introducer sheath is positioned and guiding catheter is conducted in proximal MPV ("below" the thrombus). Oval shaped defect of filling, due to thrombus is outlined. 


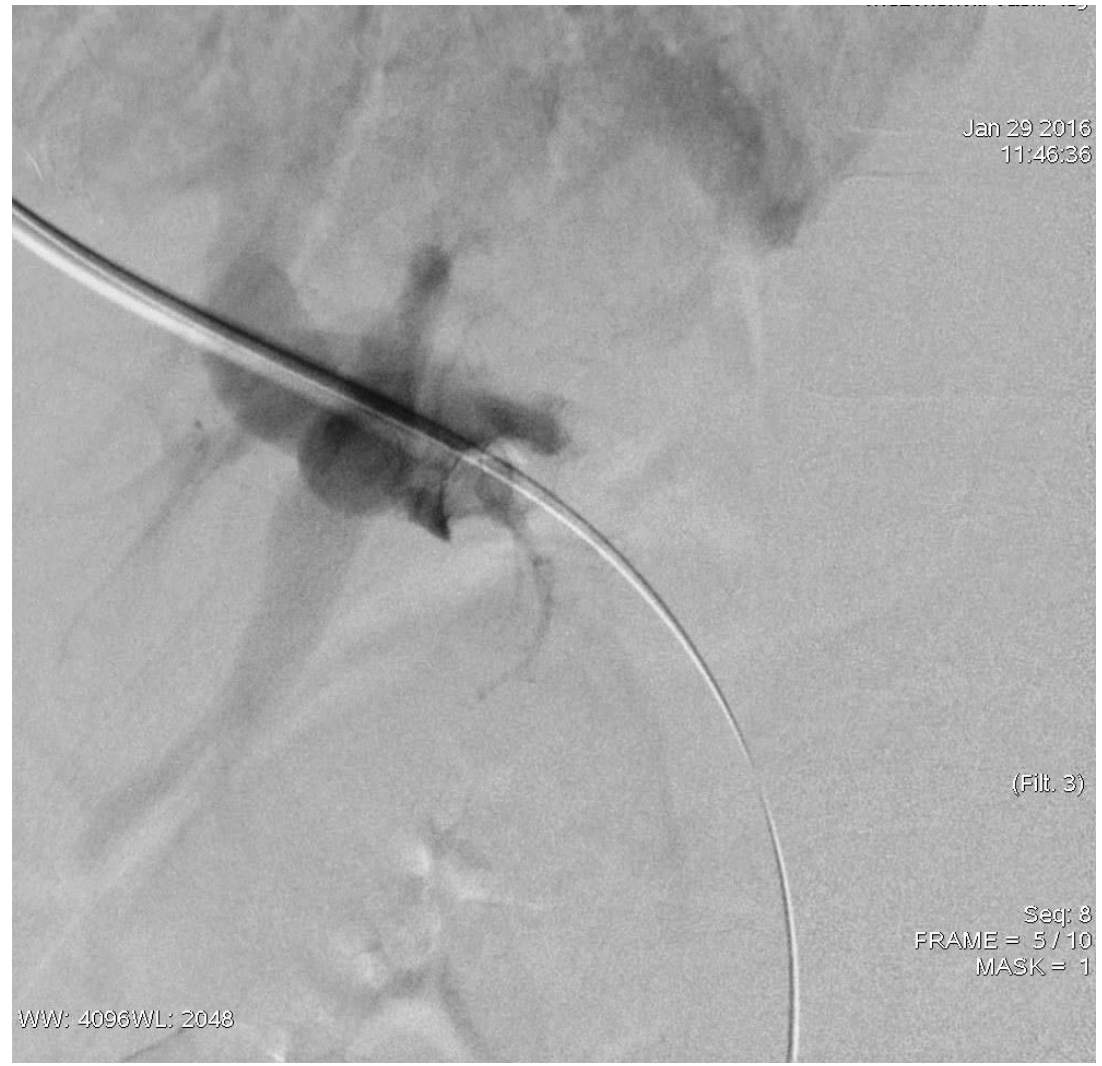

Figure 7. VesOpen procedure. Guidewire is conducted in SMV. Portography is performed using introducer sheath side port.

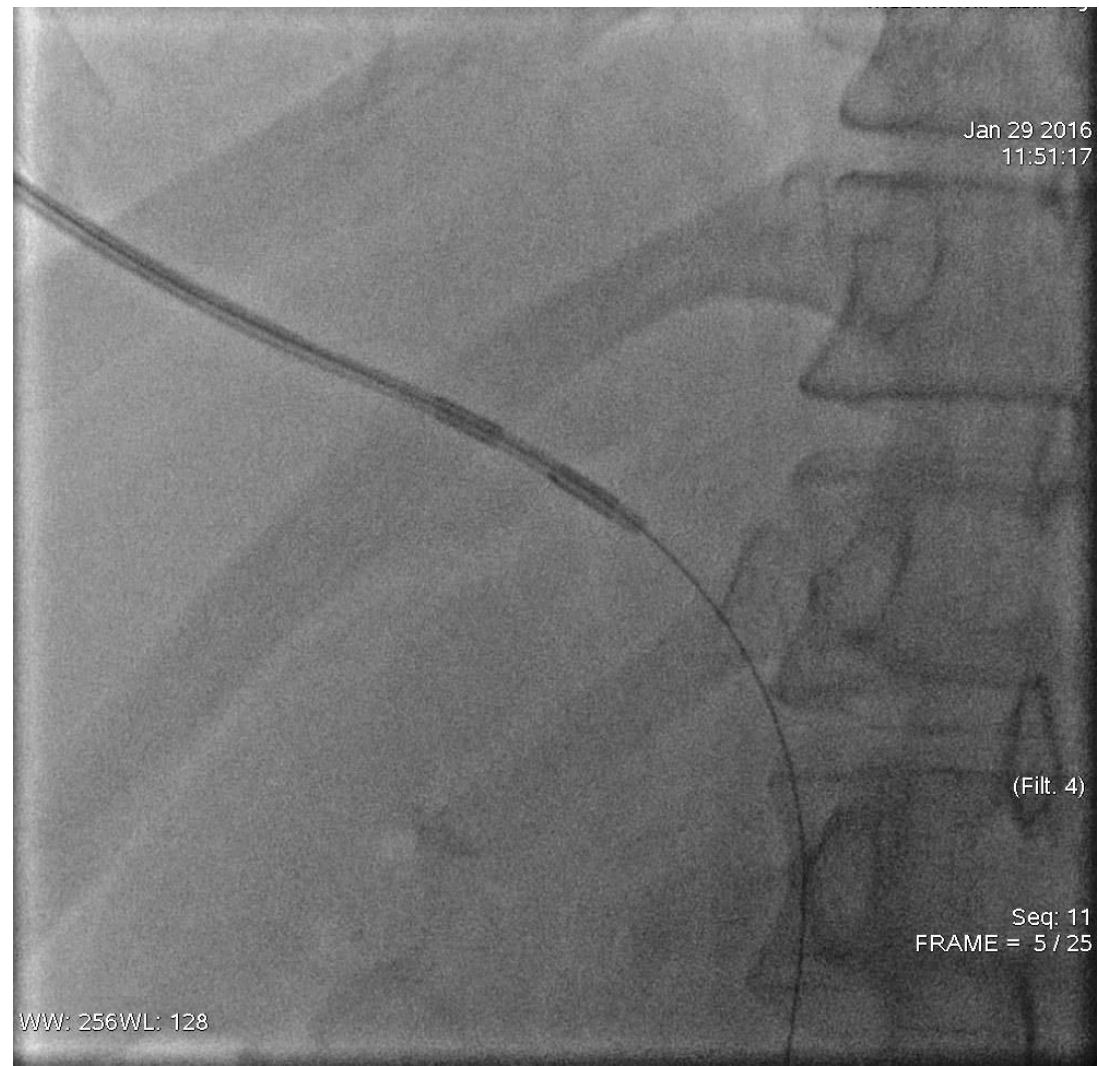

Figure 8. VesOpen procedure. Bipolar endoluminal RF device is positioned for thrombus processing. 


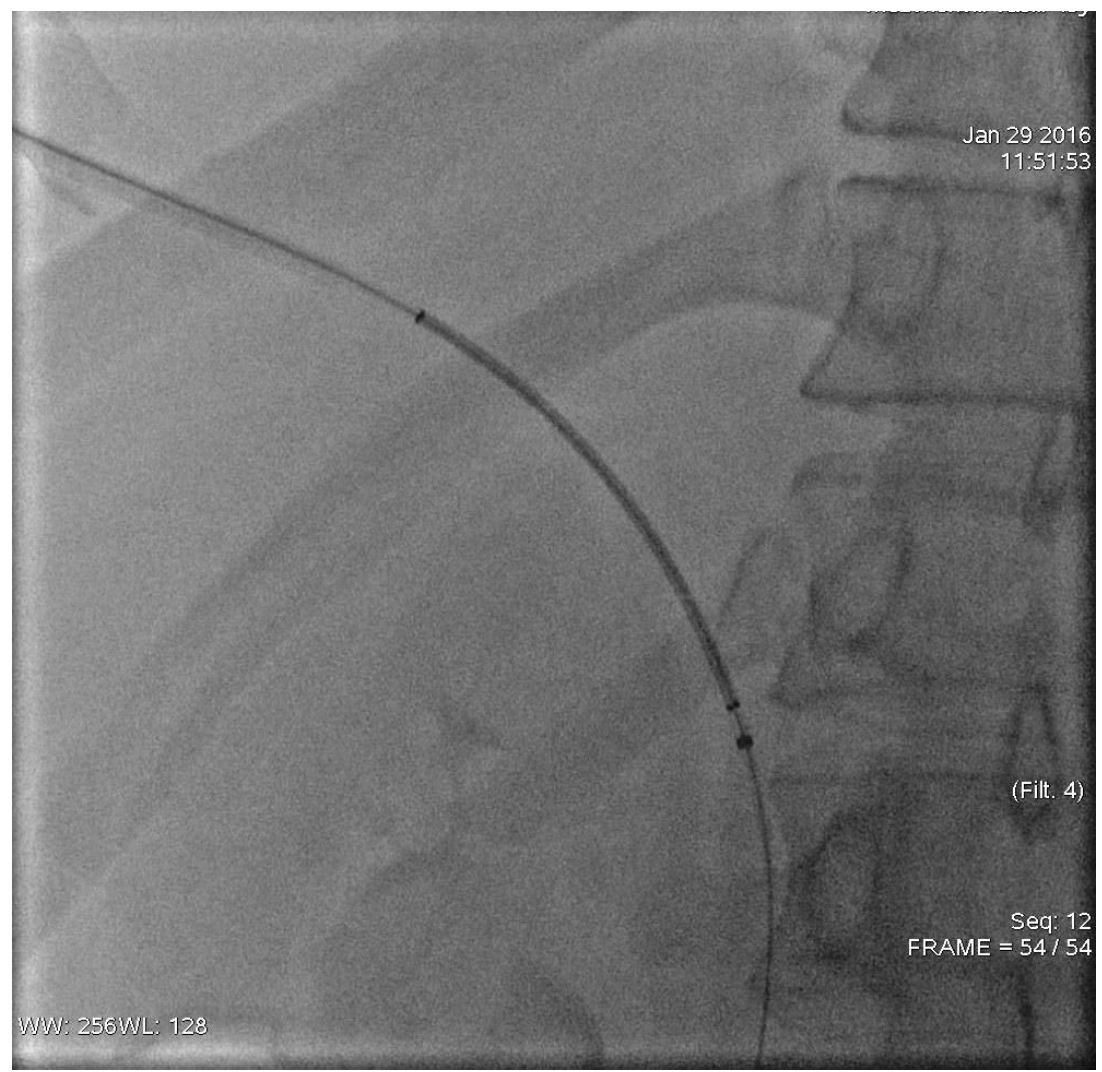

Figure 9. VesOpen procedure. Self-expanding metal stent delivery device is positioned.

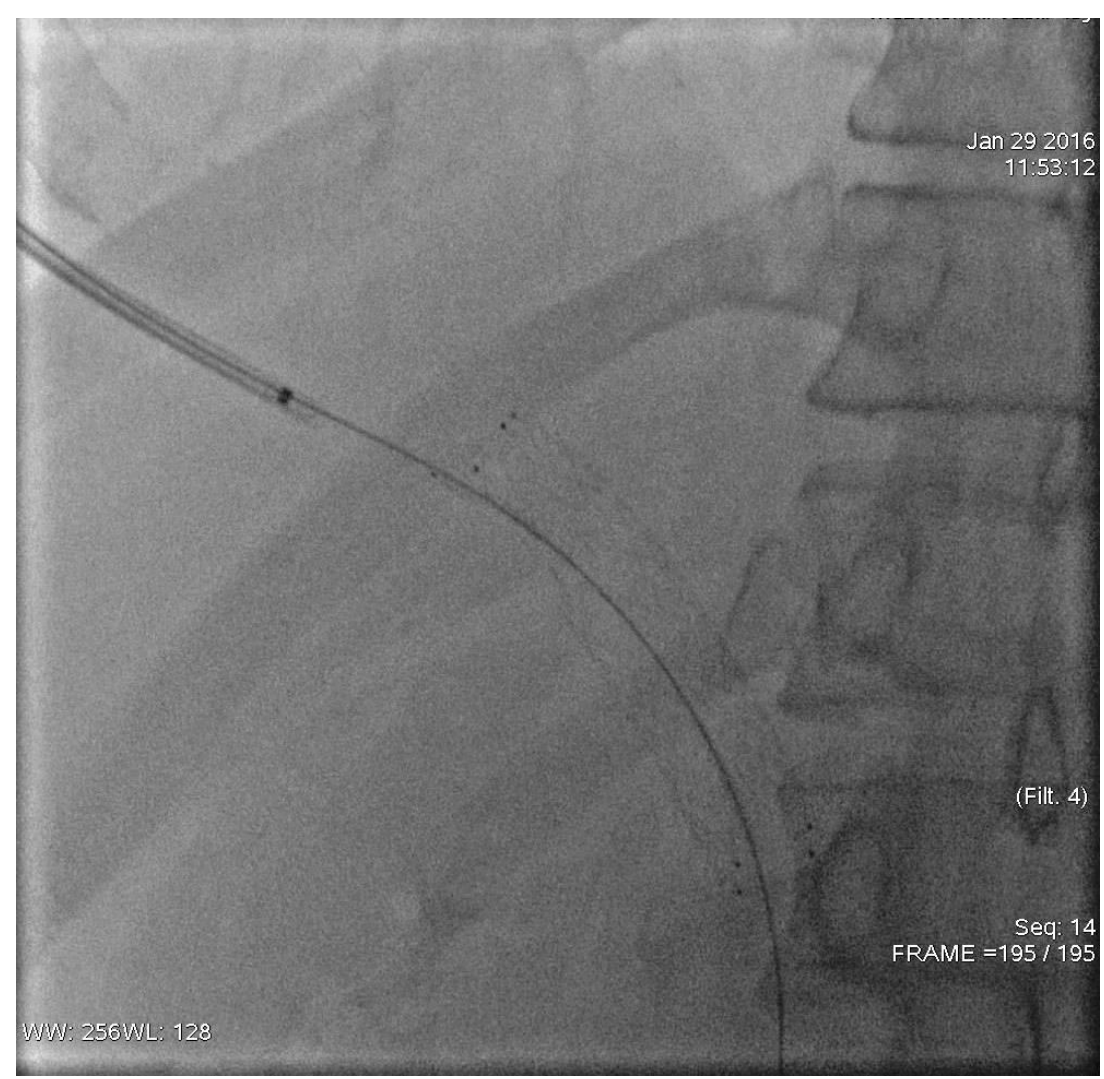

Figure 10. VesOpen procedure. Stent is released. 


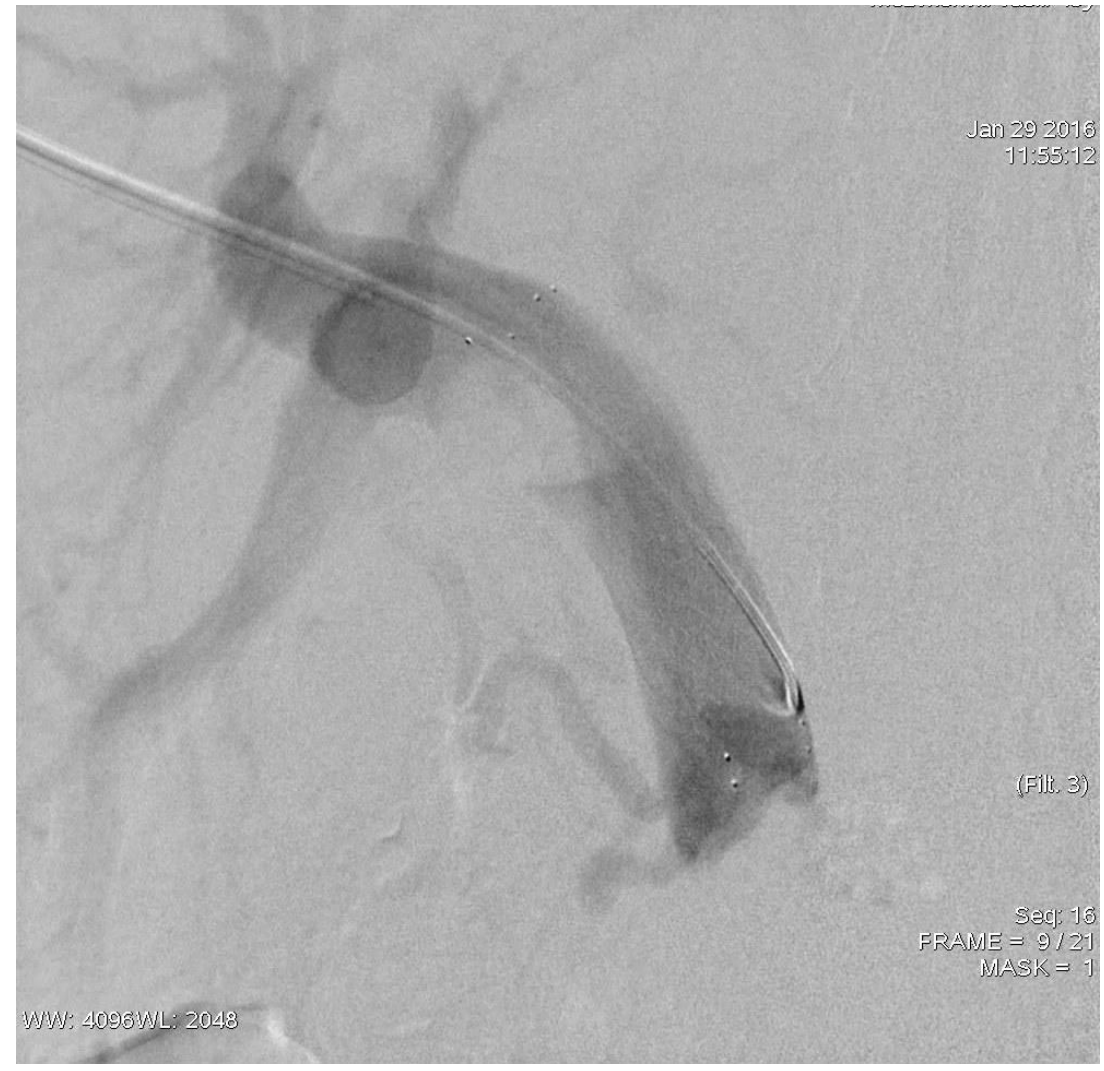

Figure 11. Portography after VesOpen procedure completion - the restored portal flow is documented

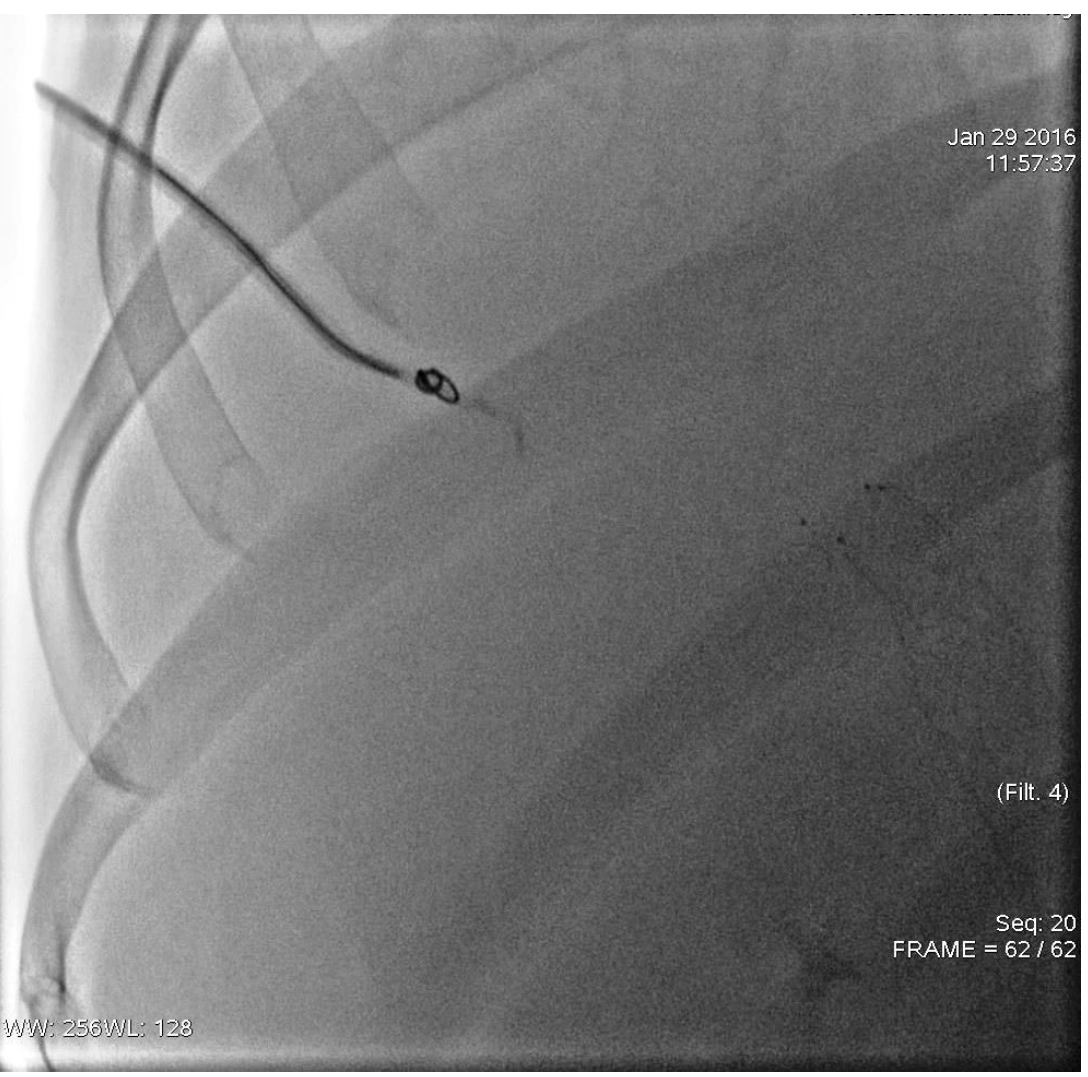

Figure 12. VesOpen procedure. Working thrack coiling for bleeding prevention. 


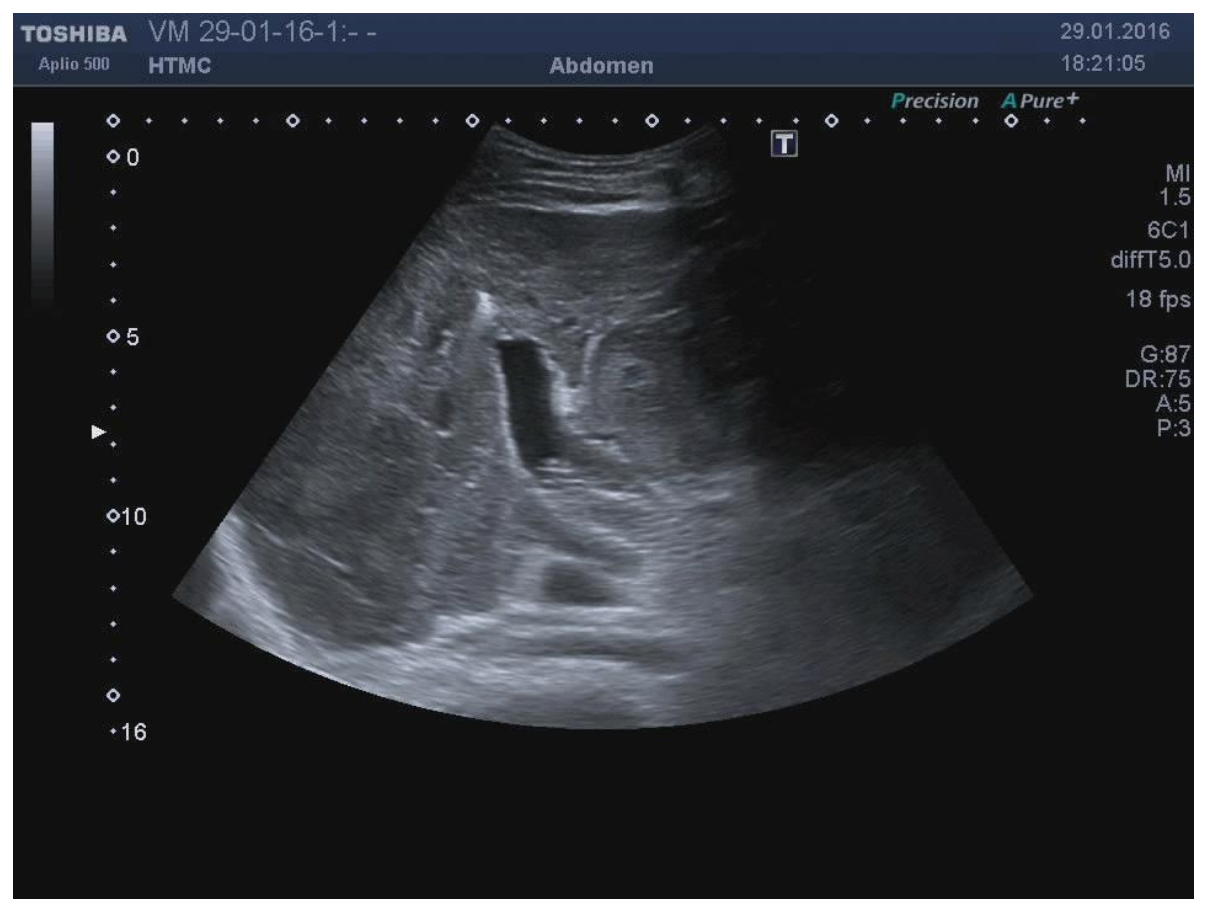

Figure 13. US after VesOpen procedure. Stent in PV and coil in hepatic parenchyma are seen

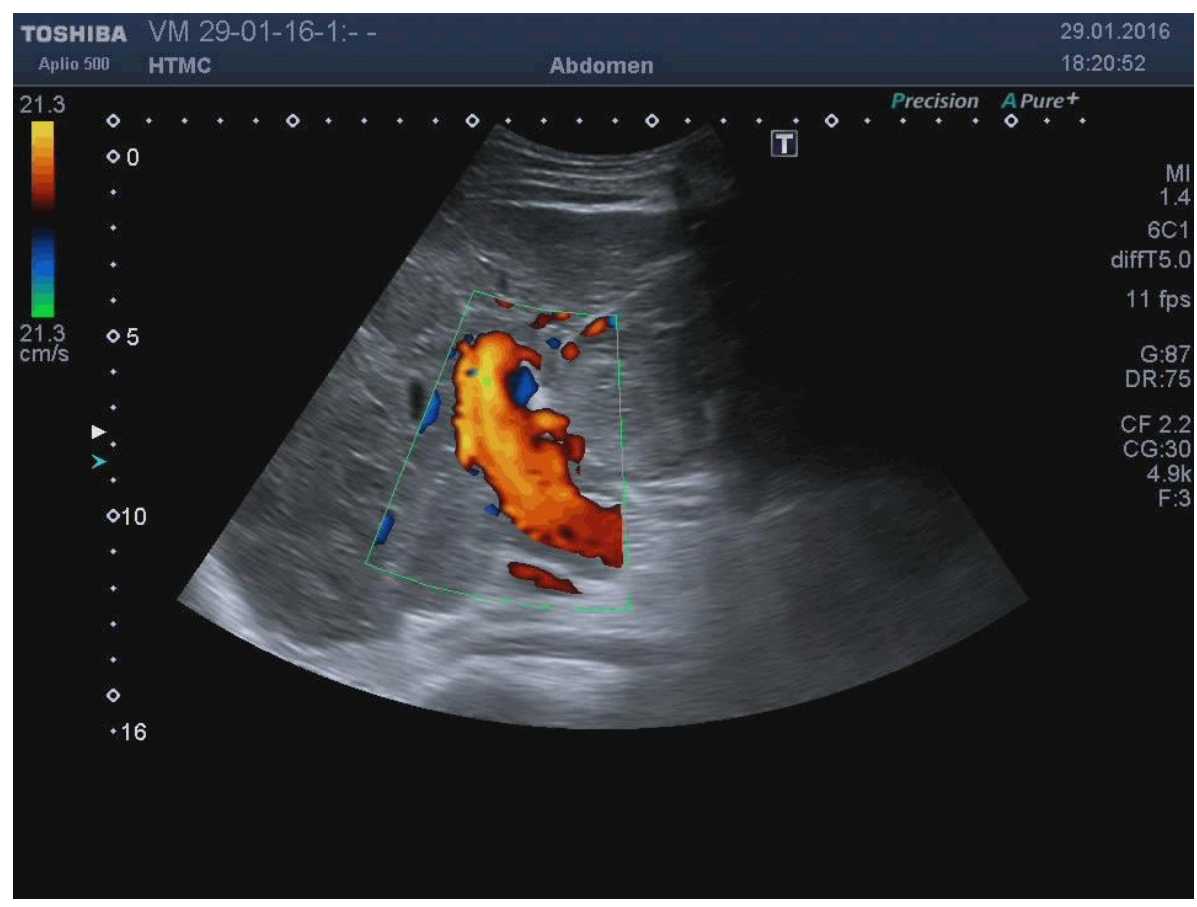

Figure 14. US after VesOpen procedure. Color Doppler documents the restored flow in MPV

Figures 15-30. Patient K. Patient with history of consecutive cholelithiasis, choledocholithiasis, pancreatitis and PV thrombosis; patient was referred to IR department because of jaundice and cholangitis onset and underwent percutaneous transhepatic biliary drainage (PTBD). After cholangitis and jaundice successful elimination patient was attempted to perform Balloon Assisted Percutaneous Descending Litholapaxy (BAPDL) for stone removal using PTBD fistula. The porto-biliary fistula was accidentally revealed on the BAPDL; the procedure has been quit and big diameter external-internal biliary drain has been placed. No hemobilia was documented after BAPDL attempt and PV stenting was performed to restore PV patency and integrity. 


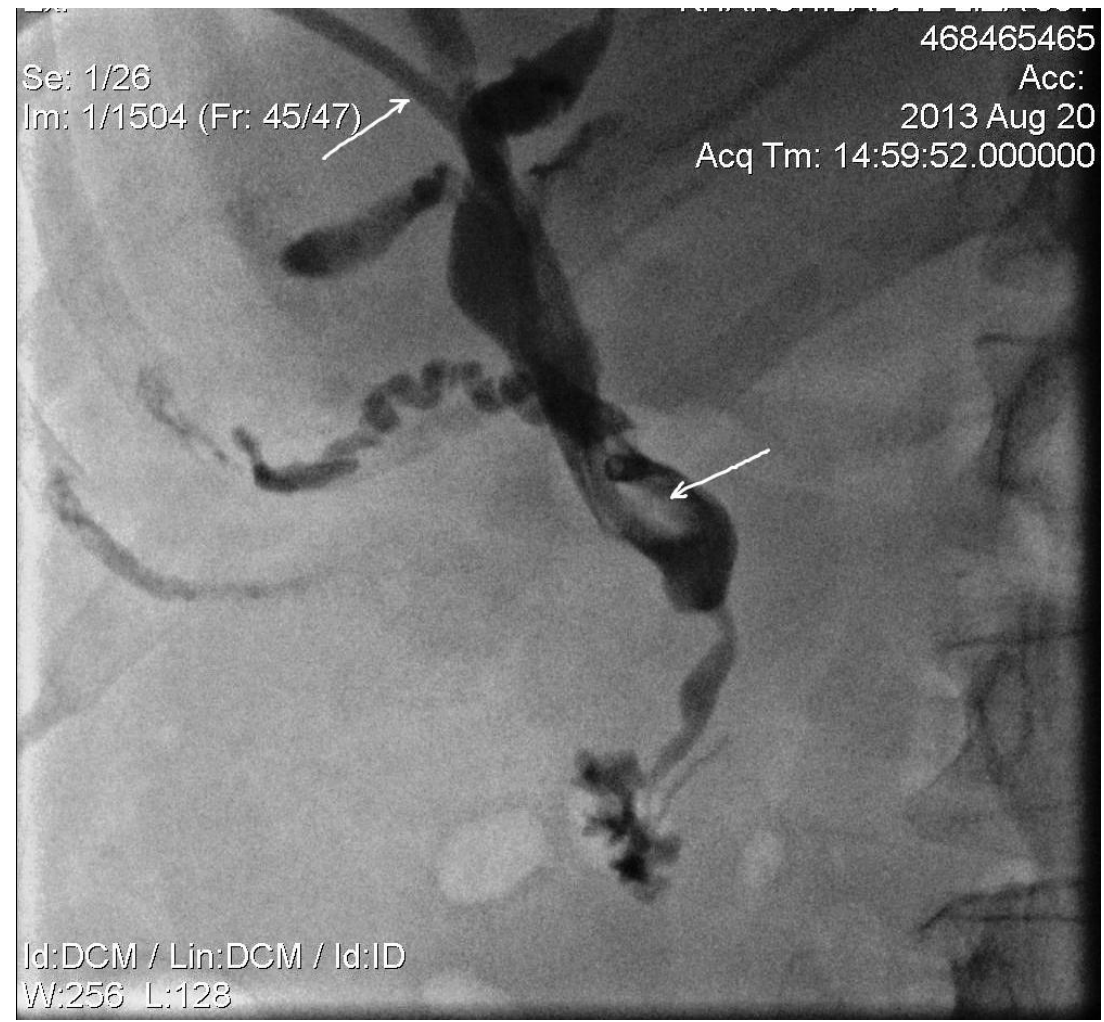

Figure 15. BAPDL procedure. Contrast is injected by PTBD catheter; arrows identify the drainage catheter and CBD stone

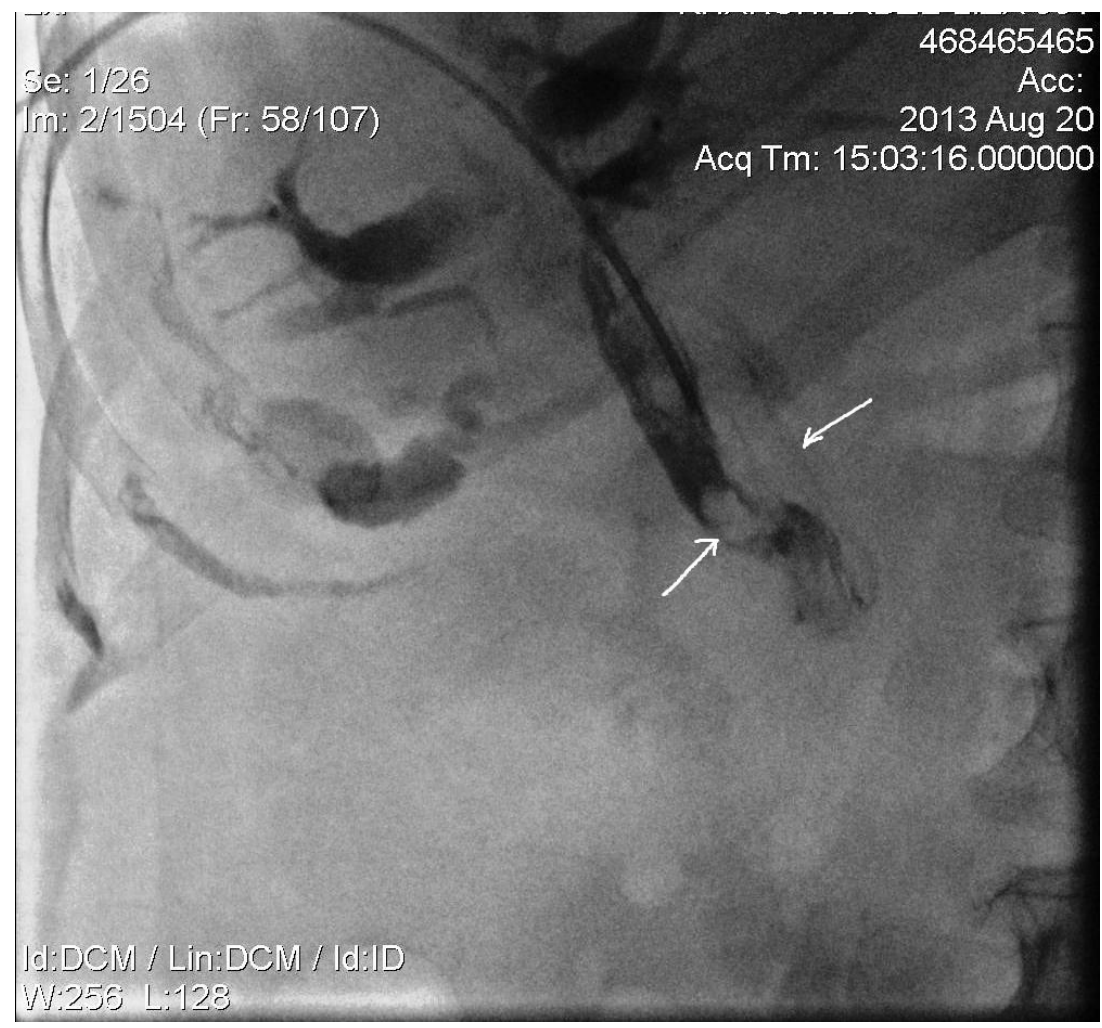

Figure 16. BAPDL procedure. Drainage catheter is removed and contrast injected by $5 \mathrm{Fr}$ diameter guiding catheter. Arrows identify the CBD stone and accidentally revealed contrast extravasation into PV. 


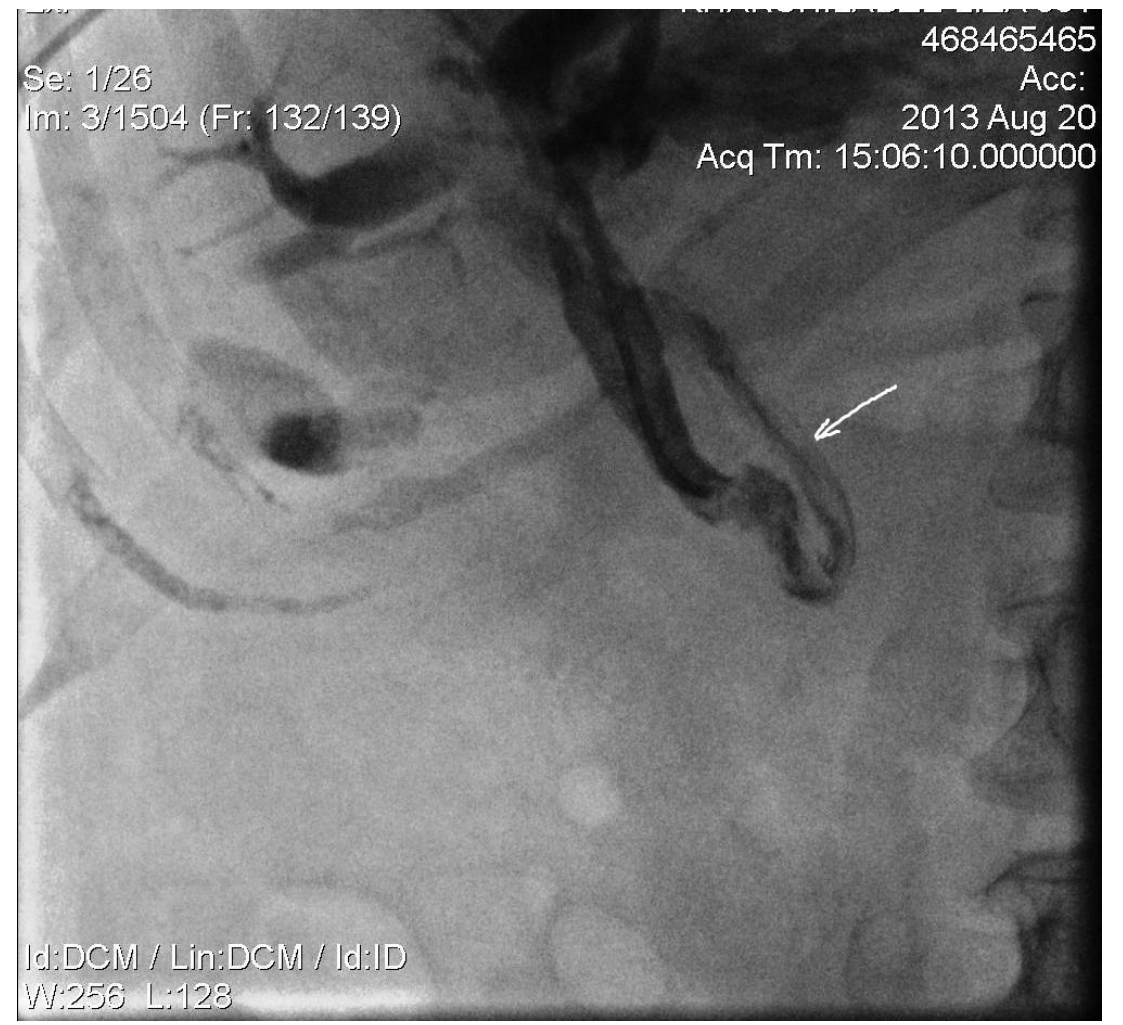

Figure 17. BAPDL procedure. Contrast injection from biliary tree shows PV (arrow)- fistula is revealed

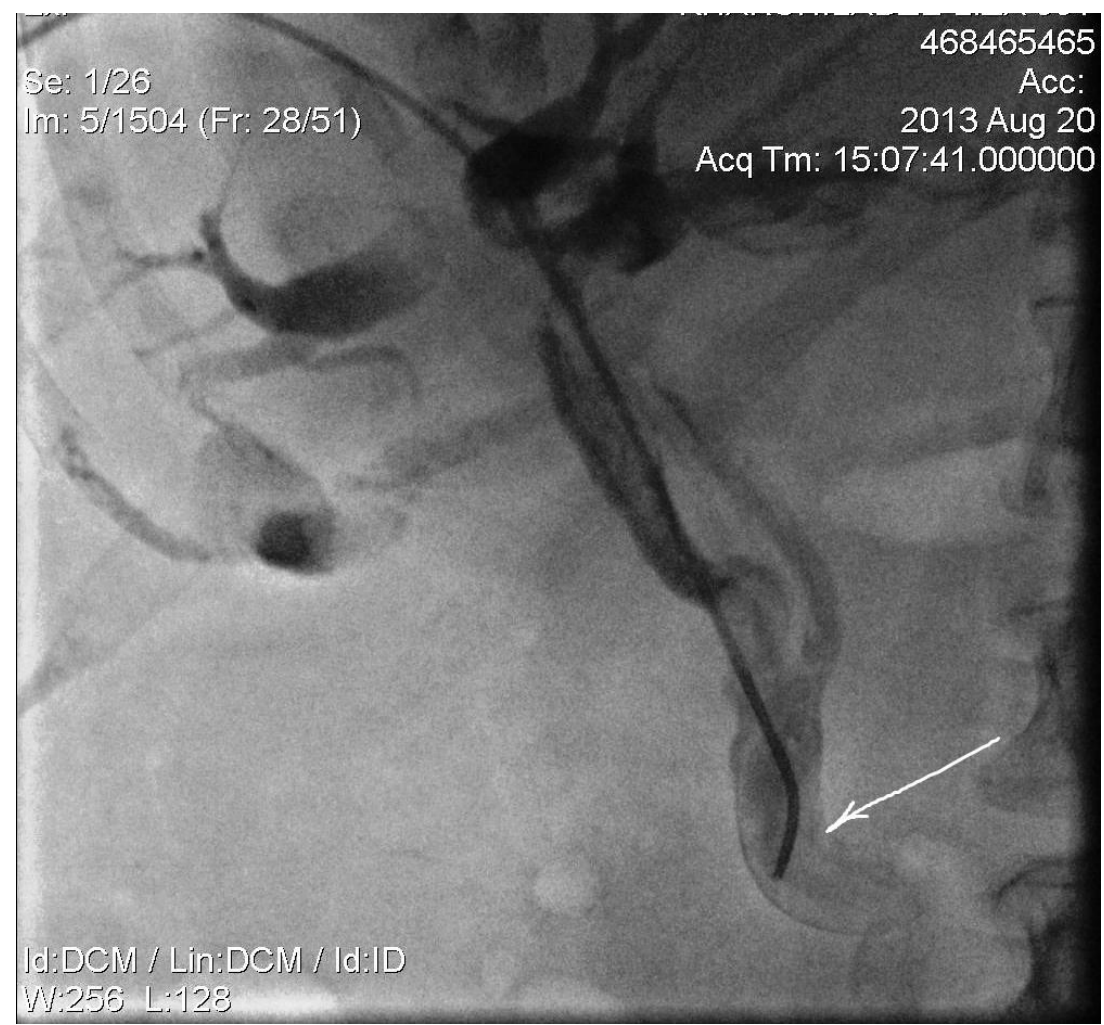

Figure 18. BAPDL procedure. When advanced, the guiding catheter enters the MPV(arrow) 

Thrombolysis, Stenting, Endoluminal RFA \& Angioplasty by Balloon or Stenting

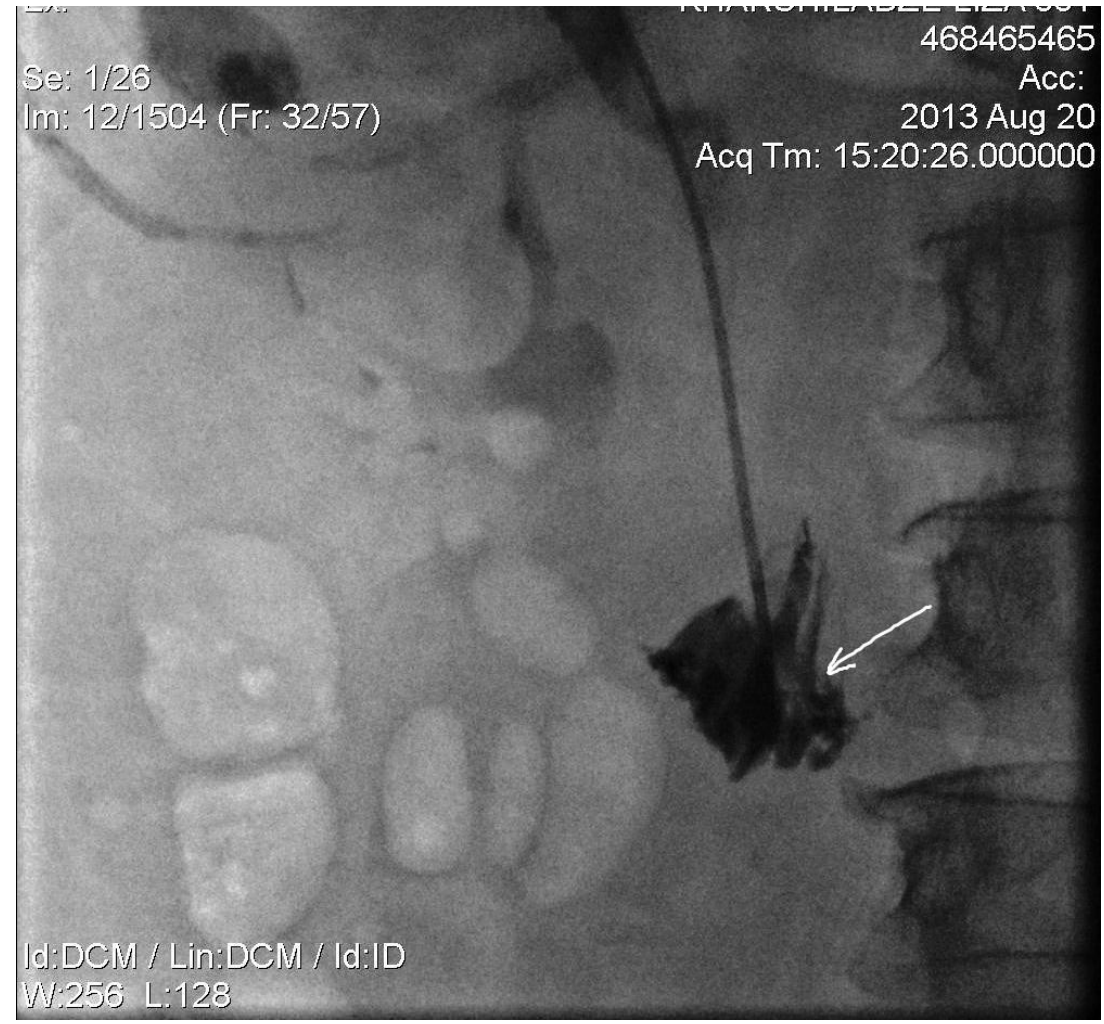

Figure 19. BAPDL procedure. The guiding catheter introduction back into CBD and duodenum (arrow) was technically very challenging

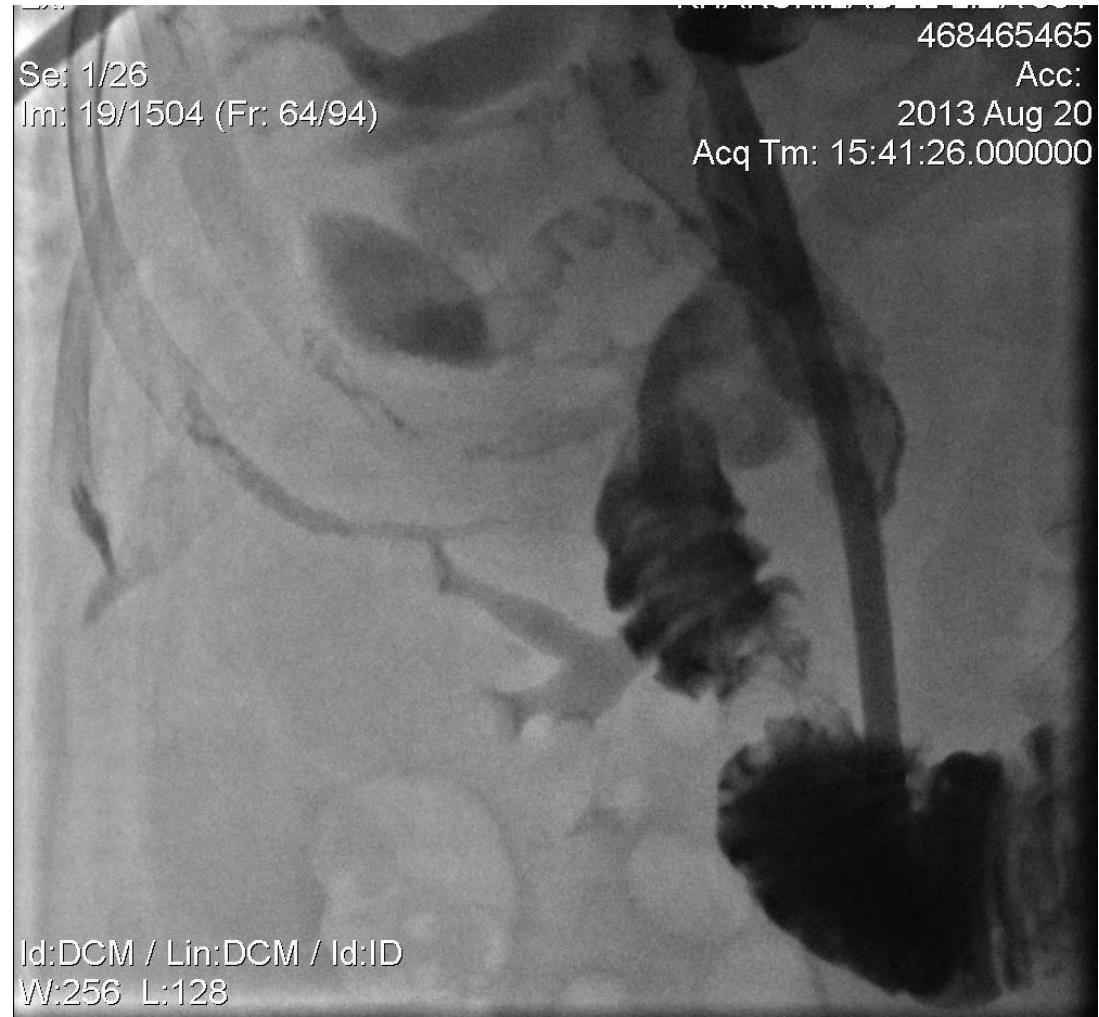

Figure 20. BAPDL procedure. The procedure has been quit and big diameter external-internal drain was positioned. 


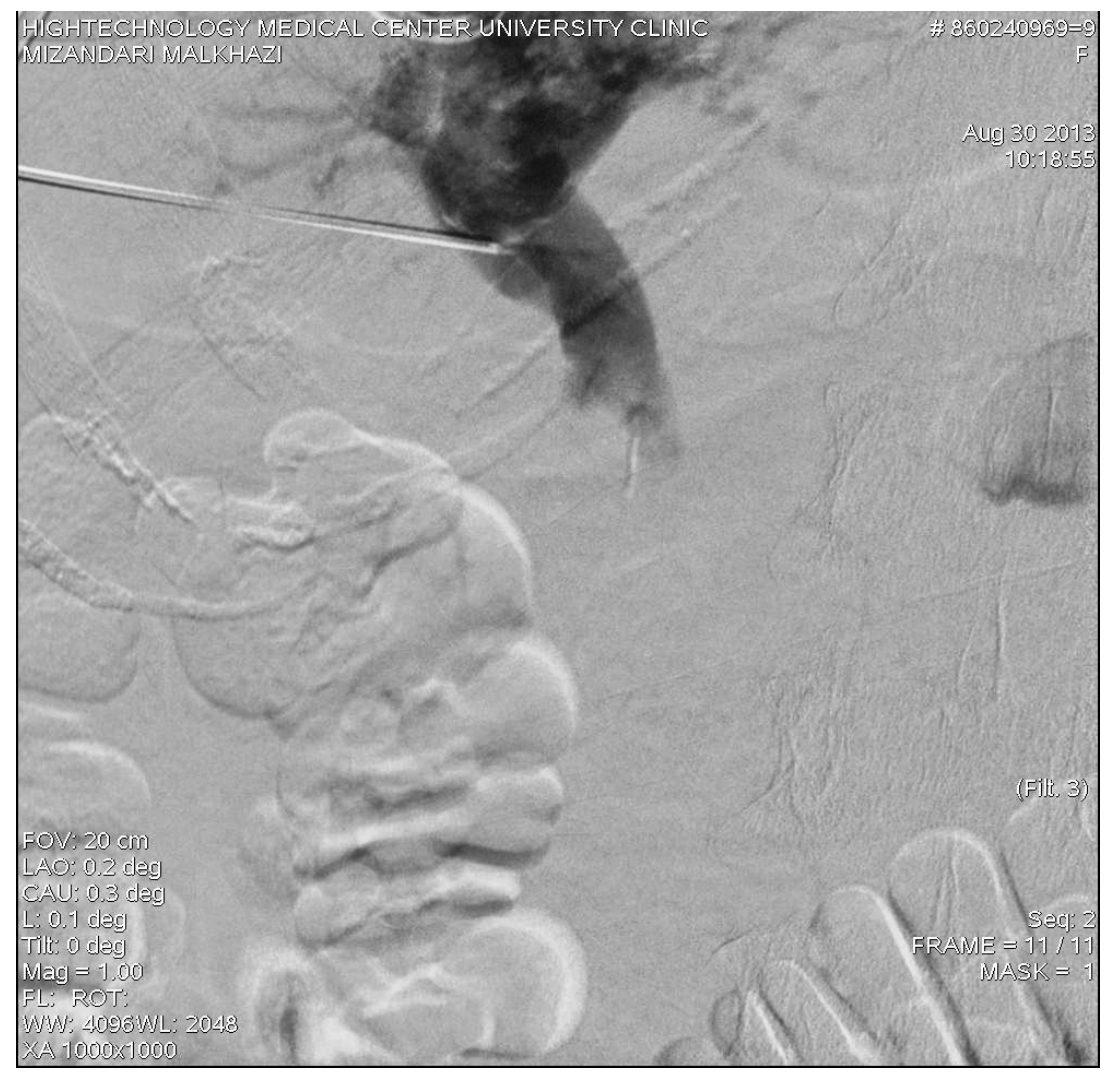

Figure 21. PV stenting procedure. Portography is performed by $18 \mathrm{G}$ diameter needle, advanced inro PV under real-time US guidance

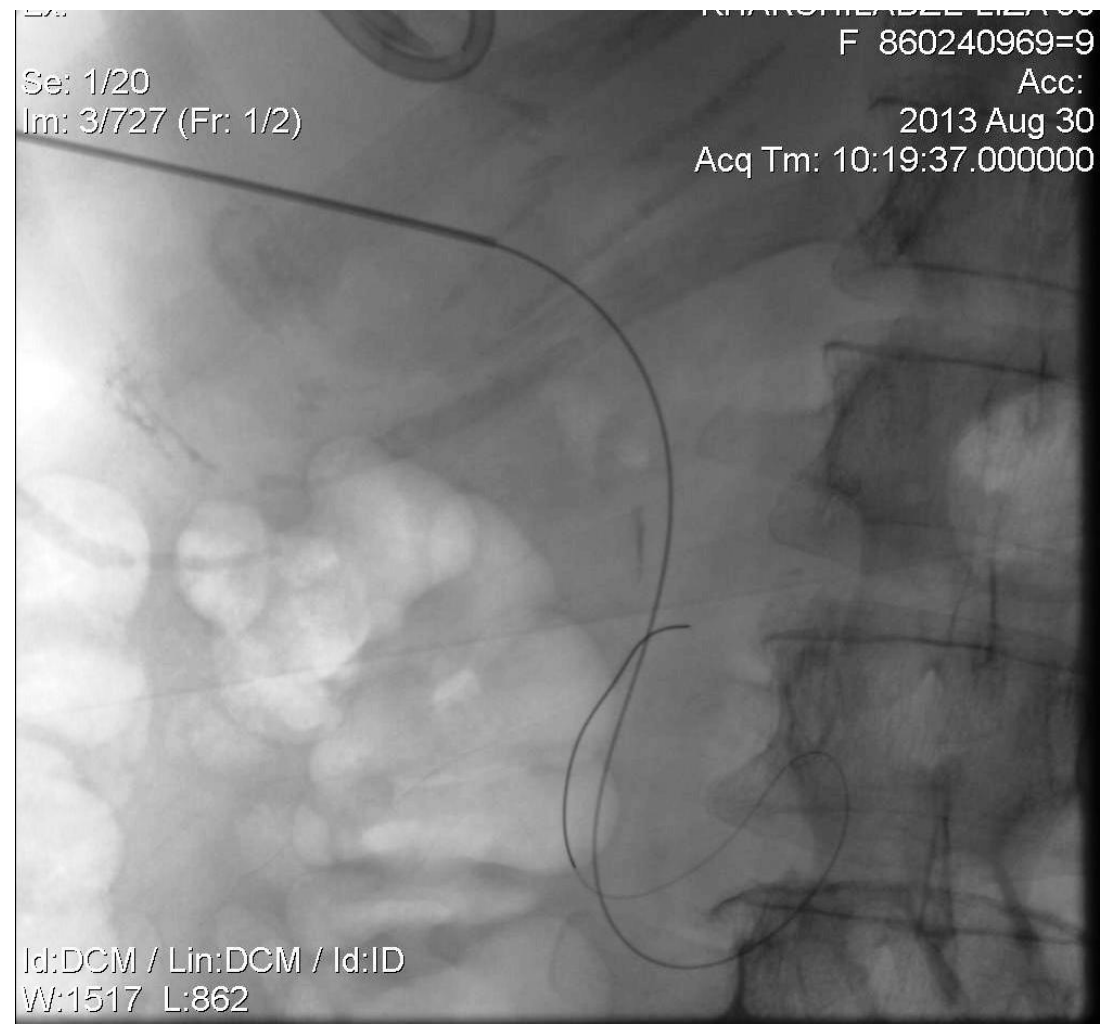

Figure 22. PV stenting procedure. The wire is successfully conducted into PV confluence. 

Thrombolysis, Stenting, Endoluminal RFA \& Angioplasty by Balloon or Stenting

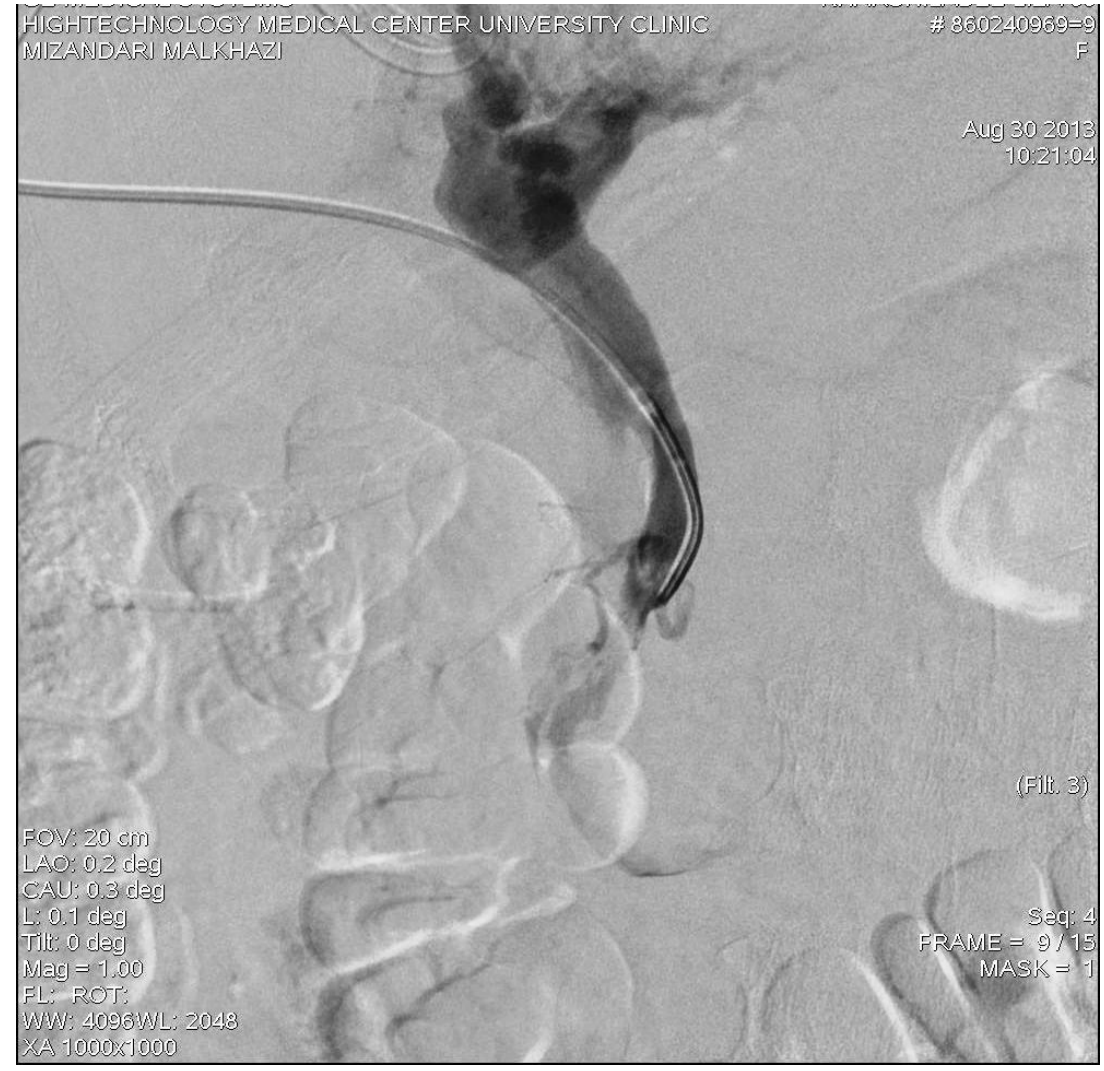

Figure 23. PV stenting procedure. 5Fr diameter guiding catheter is advanced according the wire

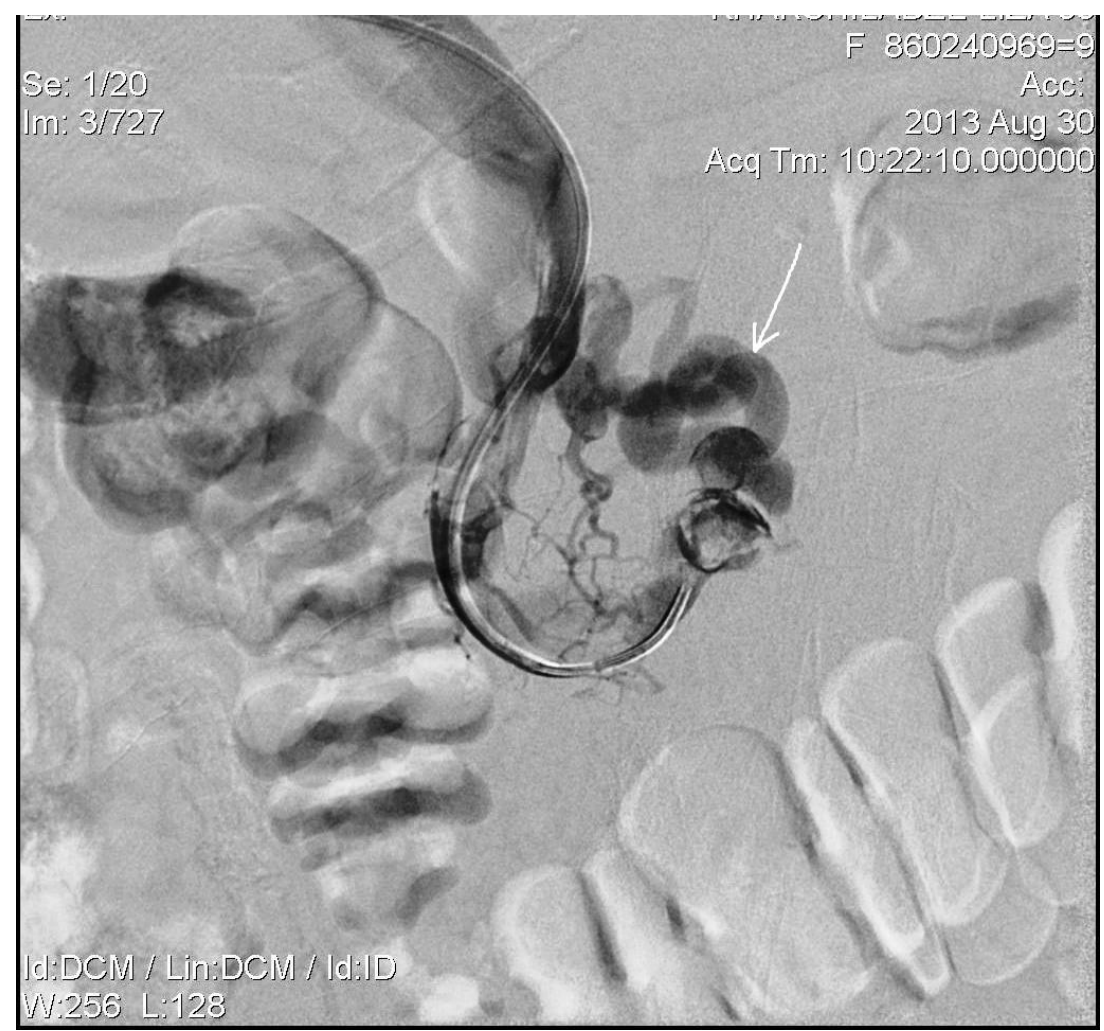

Figure 24. PV stenting procedure. Guiding catheter is advanced into the PV confluence; contrast injection shows the collateral PV flow(arrow) 


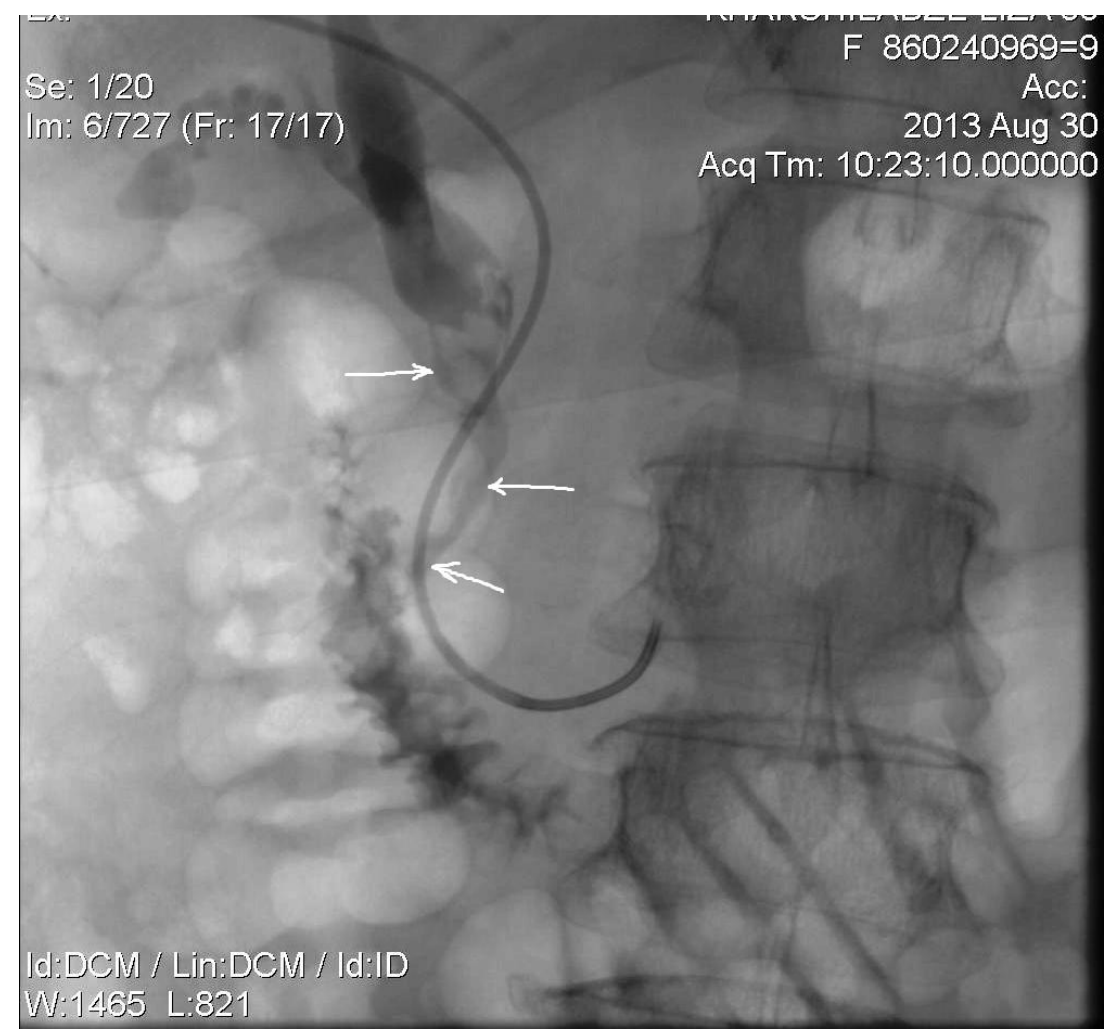

Figure 25. PV stenting procedure. Guiding catheter is in PV; contrast injection from PTBD drain opacifies the CDB (arrows)

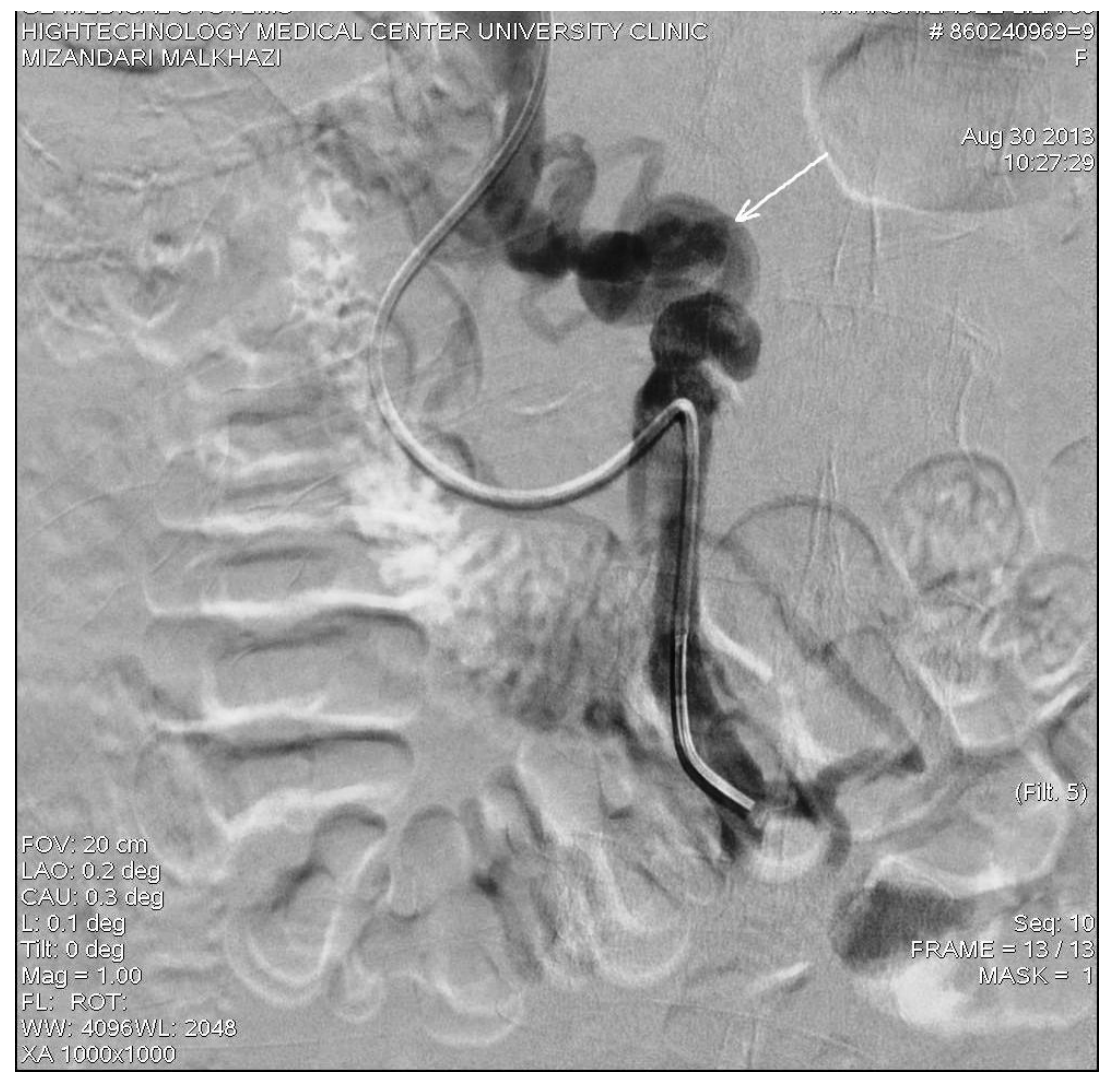

Figure 26. PV stenting procedure. Guiding catheter tip is in SMV; PV collateral flow - arrow. 

Thrombolysis, Stenting, Endoluminal RFA \& Angioplasty by Balloon or Stenting

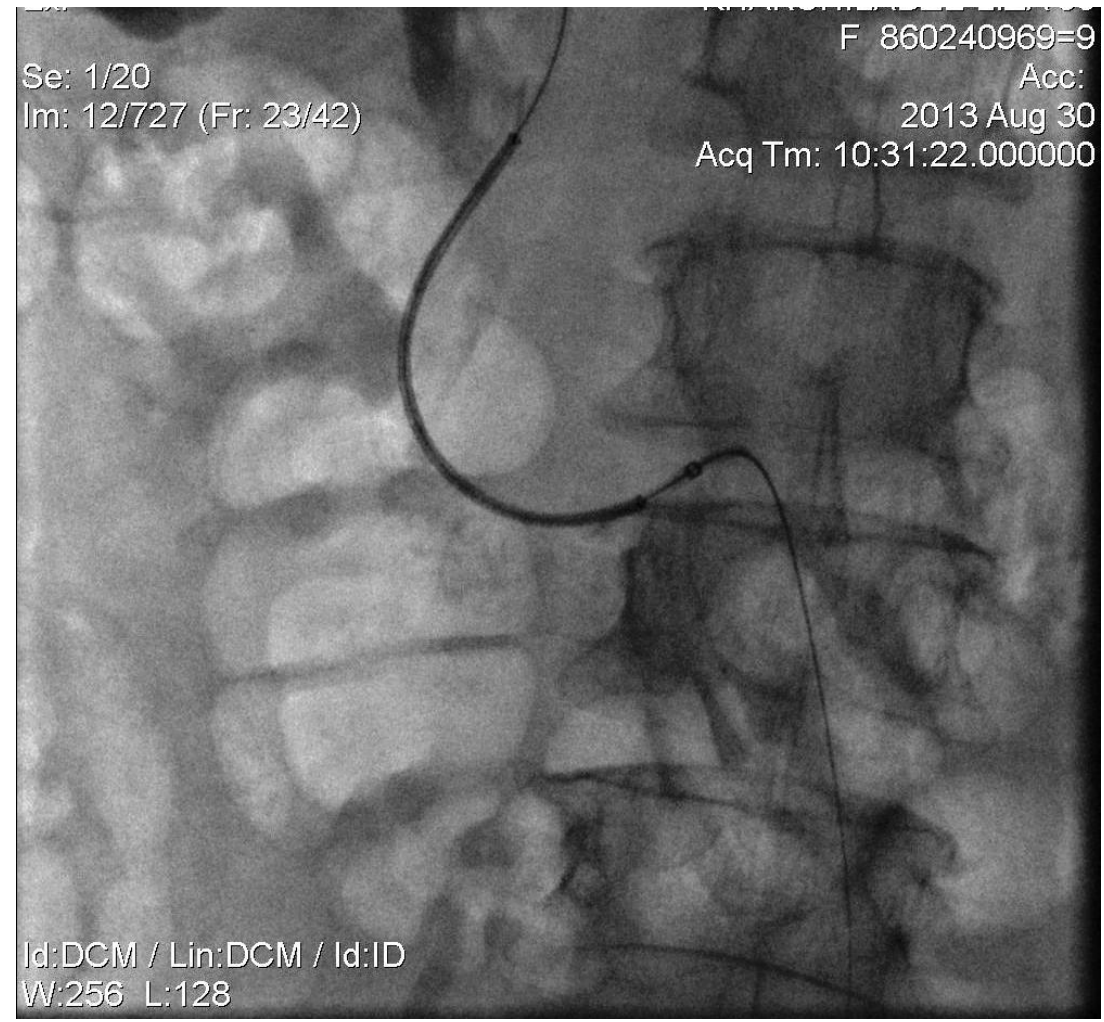

Figure 27. PV stenting procedure. Self expanding metal stent delivery device is positioned

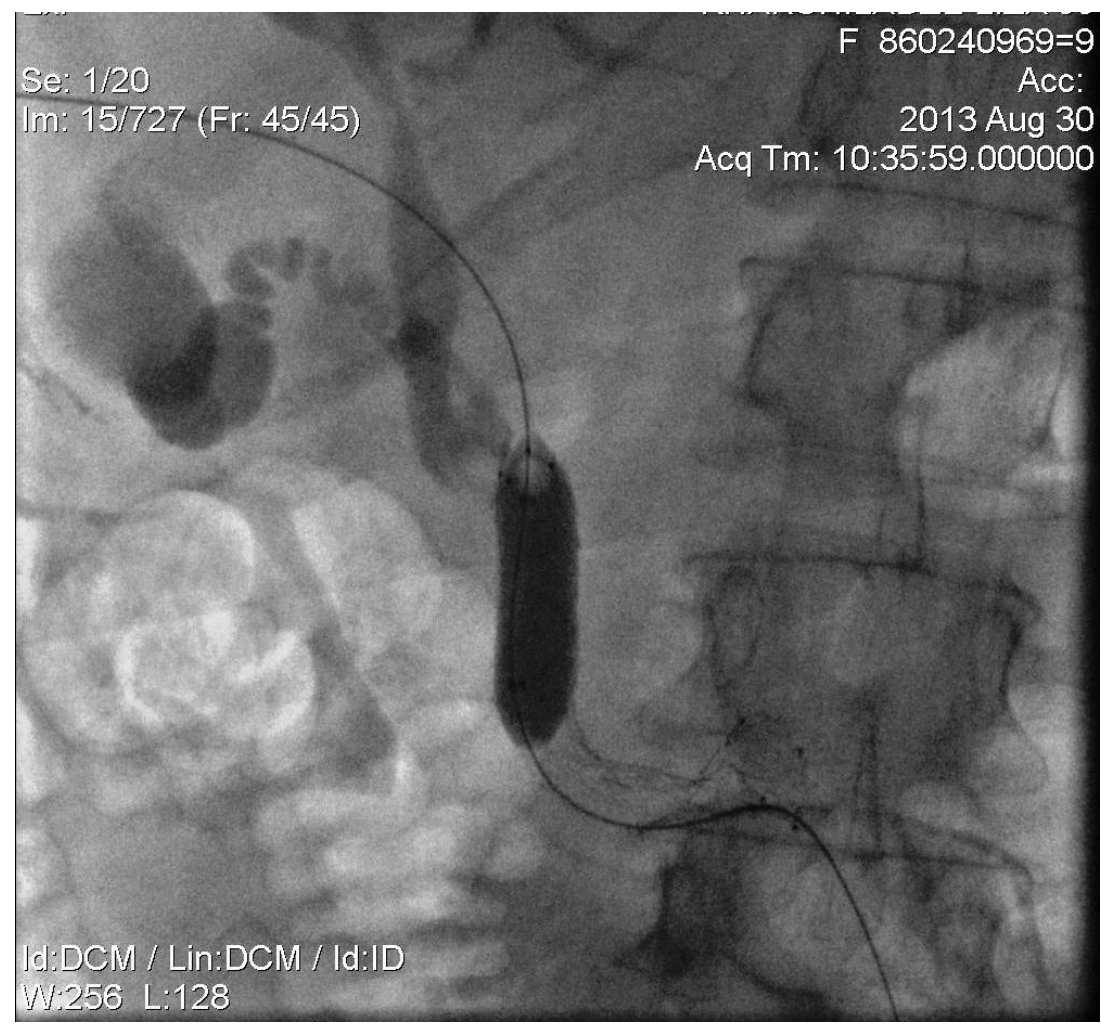

Figure 28. PV stenting procedure. Stent is released and postdilated 


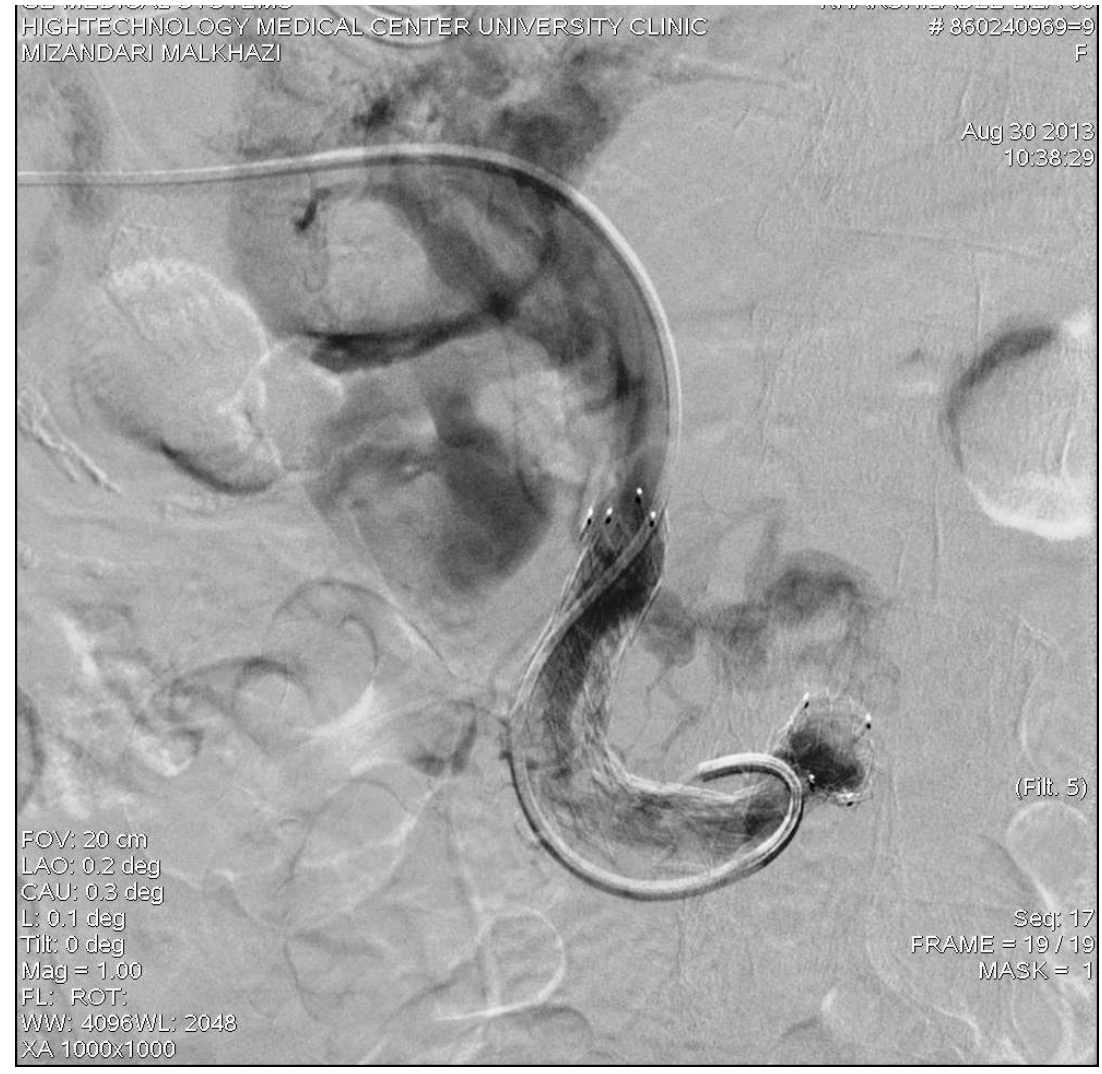

Figure 29. PV stenting procedure. Released stent in PV thrombosis and fistula segment

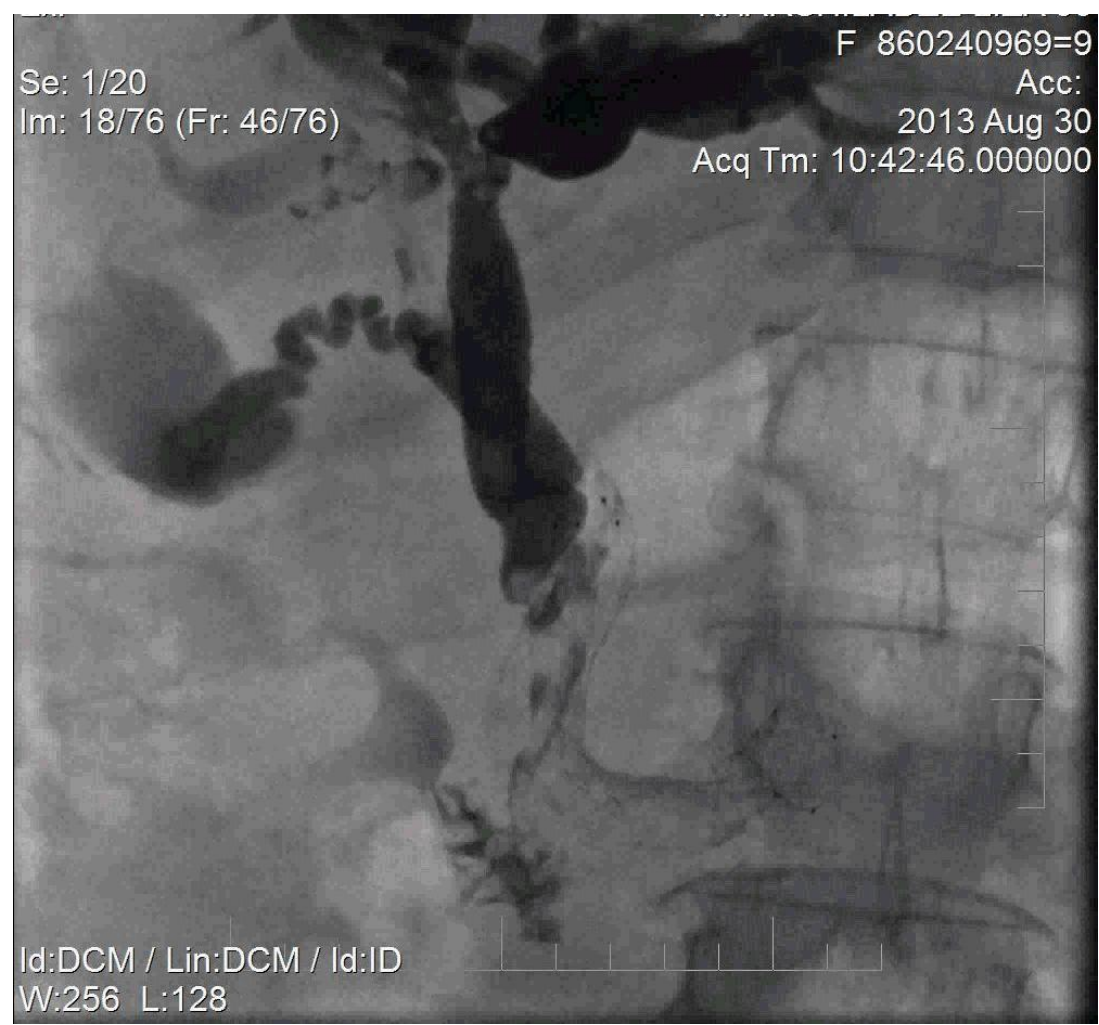

Figure 30. PV stenting procedure. No more fistula is detected by contrast injection via PTBD catheter 


\section{REFERENCES}

[1] Yogesh K. Chawla, Vijay Bodh Portal vein thrombosis. Clin Exp Hepatol. 2015 Mar; 5(1): 22-40. Published online 2015 Jan 6. doi: 10.1016/j.jceh.2014.12.008

[2] Llovet JM, Burroughs A, Bruix J (2003) Hepatocellular carcinoma. Lancet 362(9399):1907-1917

[3] Bruix J, Sherman M (2005) Management of hepatocellular carcinoma. Hepatology 42(5):1208-1236

[4] Gunven P (2008) Liver embolisation in oncology: a review. Med Oncol 25:1-11

[5] Bruix J, Sala M, Llovet JM (2004) Chemoembolization for hepatocellular carcinoma. Gastroenterology 127(5 Suppl 1):S179-S188

[6] Mizuno T, Ebata T, Yokoyama Y, Igami T, Sugawara G, Mori Y, Suzuki K, Nagino M Percutaneous transhepatic portal vein stenting for malignant portal vein stenosis secondary to recurrent perihilar biliary cancer. J Hepatobiliary Pancreat Sci. 2015 Jun 17. doi: 10.1002/jhbp. 265

[7] Ulrich Grosse, Klaus Brechtel, Dominik Ketelsen, Roland Syha, Gerd Grözing er, Fabian Springer and Christoph Thomas Portal vein recanalization and embolization of the transsplenic puncture tract using an Amplatzer vascular plug: a case report BMC Research Notes (2015) 8:193 DOI 10.1186/s13104-015-1138-4

[8] Mark R. Werley, M.D. and John Briguglio, M.D Transjugular Intrahepatic Portosystemic Shunt (TIPS ): A Clinical and Procedural Review The Journal of Lancaster General Hospital, Winter 2013118 Vol. 8 - No. 4

[9] Kangshun Zhu, MD, PhD, Xiaochun Meng, MD, PhD, Bin Zhou, MD, Jiesheng Qian, MD, Wensou Huang, MD, Meihai Deng, MD, and Hong Shan, MD, PhD Percutaneous Transsplenic Portal Vein Catheterization: Technical Procedures, Safety, and Clinical Applications J VascIntervRadiol2013;24:518-527 http://dx.doi.org/10.1016/j.jvir.2012.12.028
[10] Lazoura O, Zacharoulis D, Kanavou T et al (2011) A novel experimental animal model of arterial stenosis based on endovascular radiofrequency energy application. J Invest Surg 24(3):123-128

[11] Brown A, Malden E, Kugelmas M, Kortz E Diagnosis of pancreatic duct-portal vein fistula; a case report and review of the literature. J Radiol Case Rep. 2014 Mar 1; 8(3):31-8. doi:10.3941/jrcr.v8i3.1552.

[12] Kim HS, Patra A, Khan J, Arepally A, Streiff MB Transhepatic catheter-directed thrombectomy and thrombolysis of acute superior mesenteric venousthrombosis. J Vasc Interv Radiol. 2005 Dec;16(12):1685-91

[13] Agarwal AK, Raj Kumar K, Agarwal S, Singh S Significance of splenic vein thrombosis in chronic pancreatitis Am J Surg. 2008 Aug;196(2):149-54. doi: 10.1016/j.amjsurg.2007.07.03 9.

[14] Chaitowitz IM, Heng R, Bell KW Management of iatrogenic porto-biliary fistula following biliary stent. Australas Radiol. 2007 Dec;51 Suppl:B316-8

[15] Peynircioglu B, Cwikiel W Utility of stent-grafts in treatment of porto-biliary fistula. Cardiovascular Intervent Radiol. 2006 Nov-Dec; 29(6):1156-9.

[16] Ohkubo M, Takahashi K, Kishiro M et al (2004) Histological findings after angioplasty using conventional balloon, radiofrequency thermal balloon, and stent for experimental aortic coarctation. Pediatr Int 46(1):39-47

[17] Sreeram N, Townsend P, Morton DB (2000) Radiofrequency thermal balloon angioplasty in an experimental model of peripheral arterial stenosis. Int J Cardiol 74(1):25-32

[18] Thanos L, Sotiropoulou E, Tanteles S, Pomoni M, Kelekis D (2010) Direct radiofrequency ablation of portal vein tumor thrombus in continuity with hepatocellular carcinoma. J Vasc Interv Radiol 21(2):285-288

[19] Giorgio A, Di Sarno A, de Stefano G, Farella N, Scognamiglio U, de Stefano M, Giorgio V (2009) Hepatocellular carcinoma with cirrhosis: Are patients with neoplastic main portal vein invasion eligible for percutaneous radiofrequency ablation of both the nodule and the portal venous tumor thrombus? AJR Am J Roentgenol 193(4):948-954 